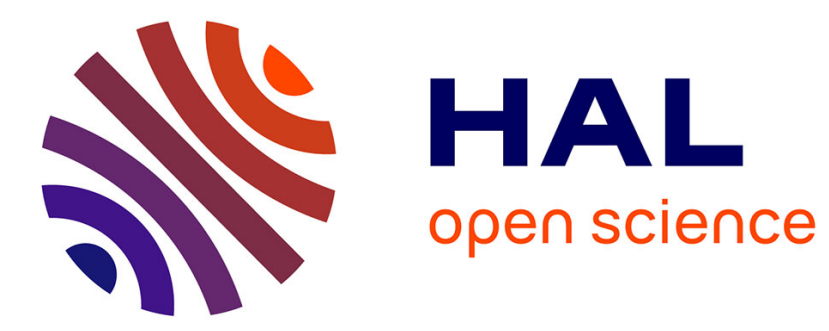

\title{
Seismic waveform modelling in a 3-D Earth using the Born approximation: potential shortcomings and a remedy
}

\author{
Mark A Panning, Yann P Capdeville, Barbara Romanowicz
}

\section{To cite this version:}

Mark A Panning, Yann P Capdeville, Barbara Romanowicz. Seismic waveform modelling in a 3-D Earth using the Born approximation: potential shortcomings and a remedy. Geophysical Journal International, 2008, 177, pp.161 - 178. 10.1111/j.1365-246X.2008.04050.x . insu-01400265

\section{HAL Id: insu-01400265}

\section{https://hal-insu.archives-ouvertes.fr/insu-01400265}

Submitted on 21 Nov 2016

HAL is a multi-disciplinary open access archive for the deposit and dissemination of scientific research documents, whether they are published or not. The documents may come from teaching and research institutions in France or abroad, or from public or private research centers.
L'archive ouverte pluridisciplinaire HAL, est destinée au dépôt et à la diffusion de documents scientifiques de niveau recherche, publiés ou non, émanant des établissements d'enseignement et de recherche français ou étrangers, des laboratoires publics ou privés. 


\title{
Seismic waveform modelling in a 3-D Earth using the Born approximation: potential shortcomings and a remedy
}

\author{
Mark P. Panning, ${ }^{*}{ }^{*}$ Yann Capdeville ${ }^{2}$ and Barbara A. Romanowicz ${ }^{3}$ \\ ${ }^{1}$ Department of Geosciences, Princeton University, Princeton, NJ 08544, USA. E-mail: mpanning@ufl.edu \\ ${ }^{2}$ Department de Sismologie, Institut de Physique du Globe de Paris, Paris, France \\ ${ }^{3}$ Berkeley Seismological Laboratory, University of California, Berkeley, CA 94720, USA
}

Accepted 2008 November 11. Received 2008 November 11; in original form 2007 September 10

\begin{abstract}
SUMMAR Y
Although the use of the first-order Born approximation for the computation of seismic observables and sensitivity kernels in 3-D earth models shows promise for improving tomographic modelling, more work is necessary to systematically determine how well such methods forward model realistic seismic data compared with more standard asymptotic methods. Most work so far has been focused on the analysis of secondary data, such as phase velocity, rather than time domain waveforms. We here compare synthetic waveforms obtained for simple models using standard asymptotic approximations that collapse the sensitivity to 3-D structure on the great circle plane and those obtained using the 3-D linear Born approximation, with accurate numerical 3-D synthetics. We find, not surprisingly, that 3-D Born more accurately models the perturbation effects of velocity anomalies that are comparable in wavelength to or are smaller than the first Fresnel zone. However, larger wavelength and amplitude anomalies can easily produce large phase delays that cause the first-order (linear) Born approximation to break down, whereas asymptotic methods that incorporate the effect of heterogeneity in the phase rather than in the amplitude of the waveform are more robust. Including a path average phase delay to the Born calculated waveforms significantly improves their accuracy in the case of long-wavelength structure, while still retaining the ability to correctly model the effect of shorter-wavelength structure. Tests in random models with structural wavelengths consistent with existing global seismic models indicate that the linear Born approximation frequently breaks down in realistic earth models, with worse misfit for first and second orbit Rayleigh and higher mode surface waveforms than the great-circle based approximations at all distances tested $\left(>20^{\circ}\right)$. For fundamental modes, the average misfit for the waveforms calculated with the linear Born formalism is quite poor, particularly for distances larger than $60^{\circ}$. The modified Born formalism consistently improves the fit relative to the linear Born waveforms, but only outperforms the great-circle based approximations for the higher mode surface waveforms. We note, however, that phase delay kernels for multitaper measurements of fundamental mode Rayleigh wave phase velocities developed from the Born approximation do not demonstrate the problems associated with the linear waveform kernels. There is general agreement with measurements and moderate improvement relative to phase delays predicted by the path-average approximation.
\end{abstract}

Key words: Surface waves and free oscillations; Seismic tomography; Theoretical seismology; Wave scattering and diffraction.

\section{INTRODUCTION}

Progress in our understanding of the seismic structure of the deep Earth has always relied on companion advances in theory and data.

\footnotetext{
* Now at: Department of Geological Sciences, University of Florida, Gainesville, FL 32605, USA.
}

In recent years there has been considerable interest in advancing global and regional scale model resolution, through the use of 'finite-frequency' kernels that move beyond ray theory infinite frequency approximations to more accurately reflect the 3-D sensitivity of seismic observables (e.g. Ritzwoller et al. 2002; Montelli et al. 2004, 2006; Yoshizawa \& Kennett 2004; Zhou et al. 2006; Boschi 2006; Takeuchi 2007). The particular theory and data set used in each of these models vary, but all of these models are 
developed with the idea that using an improved theory should result in better resolution, particularly at the finer structural scales where other theories commonly in use are expected to break down.

For large-scale global and regional scale models, most of these approaches are based on theoretical developments enabling practical application of either the first-order Born (Dahlen et al. 2000; Zhao et al. 2000; Zhou et al. 2004) or Rytov approximations (Spetzler \& Snieder 2001). However, similar theoretical approaches for waveform tomography based upon acoustic theory were applied earlier and are still in use in more local exploration settings (e.g. Woodward 1992; Brenders \& Pratt 2007). Although still a computational challenge, related methods based upon adjoint approaches have also been proposed for global scale work (Tromp et al. 2005; Liu \& Tromp 2006; Tape et al. 2007). There are also models that incorporate finite-frequency effects in a more computationally efficient manner by collapsing the 3-D sensitivity to a 2-D sensitivity along the great circle path between source and receiver ( $\mathrm{Li} \&$ Romanowicz 1995).

Most of the published global and regional scale finite-frequency models to date, like many of the tomographic models that preceded them, have been derived from body wave traveltime data (Montelli et al. 2004, 2006) and/or multimode surface wave phase and group delay measurements (Ritzwoller et al. 2002; Yoshizawa \& Kennett 2004; Boschi 2006; Zhou et al. 2006). However, there are several published whole mantle global models based in whole or in part upon time-domain waveform inversion methods (e.g. Li \& Romanowicz 1996; Mégnin \& Romanowicz 2000; Gu et al. 2003; Panning \& Romanowicz 2006; Takeuchi 2007). The theory used for the waveform modelling ranges from the computationally efficient assumption that the waveforms are sensitive only to path-averaged structure (Woodhouse \& Dziewonski 1984) to the somewhat more computationally expensive technique of reducing finite-frequency sensitivity to 2-D sensitivity along a plane (Li \& Romanowicz 1995) to fully 3-D kernels based upon the Born approximation and numerical wavefield calculations (Takeuchi 2007).

There are several reasons why a time-domain waveform modelling approach may be preferred over secondary observables such as surface wave phase delays measured in the frequency domain or body wave traveltimes measured by picks or cross-correlation. There is arguably more information contained in the full waveforms than in the secondary observables, which act to reduce the waveform to a smaller set of parameters, such as phase delay or amplitude perturbation. This is particularly important when dealing with portions of the seismograms that include interfering phases (e.g. overtone surface waves and closely arriving body wave phases such as $S$ and $S c S$ in many distance ranges), where simple phase delay measurements or cross-correlation traveltimes are more problematic. However, the challenges of using 3-D implementations of the Born approximation for time-domain waveform modelling are not the same as those for other data types, and it is important to evaluate how well such an approach actually models the waveforms we can observe.

With that in mind, further systematic evaluation of how well such approaches are able to model realistic global seismic waveforms is necessary. In this study, we evaluate some limits of applicability of the linear 3-D Born approximation for time-domain waveform modelling and see how it compares with other theoretical approaches and make suggestions for how to extend the applicability of the 3-D Born waveform kernels without requiring extensive computational capacity such as for adjoint methods. For comparison to the Born approximation applied in the context of secondary observables, we also analyse the effectiveness of 3-D Born phase delay kernels (as opposed to waveform kernels) for predicting fundamental mode Rayleigh wave phase delays.

\section{BORN APPROXIMATION}

The first-order Born approximation is a single scattering approximation, used in seismological applications to approximate the perturbed wavefield due to a small perturbation of the reference medium. The simplified derivation that follows illustrates what is meant by terming it a 'single scattering' approach.

Neglecting the effects of gravity, the homogeneous equations of motion in an elastic medium can be expressed concisely as

$\rho \partial_{t}^{2} \mathbf{u}_{0}-\nabla \cdot \mathbf{C}: \varepsilon_{0}=0$,

$\mathcal{L}_{0} \mathbf{u}_{0}=0$,

where $\mathbf{u}_{0}$ is the displacement field in the reference medium, $\mathbf{C}$ is the fourth order elastic stiffness tensor and $\varepsilon_{0}$ is the strain tensor in the reference medium. Eq. (2) simply restates eq. (1) using an integro-differential operator $\mathcal{L}_{0}$. The elastic response of the medium can then be determined by placing a forcing term on the right-hand side.

A small perturbation to the reference medium can be approximated as a small perturbation to both the operator $\left(\mathcal{L}_{0} \rightarrow \mathcal{L}_{0}+\delta \mathcal{L}\right)$ and the wavefield $\left(\mathbf{u}_{0} \rightarrow \mathbf{u}_{0}+\delta \mathbf{u}\right)$,

$\left(\mathcal{L}_{0}+\delta \mathcal{L}\right)\left(\mathbf{u}_{0}+\delta \mathbf{u}\right)=0$,

$\mathcal{L}_{0} \delta \mathbf{u}=-\delta \mathcal{L} \mathbf{u}_{0}+\mathcal{O}\left(\delta^{2}\right)$,

where eq. (4) defines the first-order Born approximation upon neglecting the higher order perturbation terms. This sets up a method for calculating the perturbation to the reference wavefield, $\delta \mathbf{u}$, by considering forcing terms in the original equations of motion calculated by considering the effect perturbed medium, $\delta \mathcal{L}$, operating on the reference wavefield, $\mathbf{u}_{0}$. We can model the perturbed wavefield by integrating over the entire perturbed medium considering the forcing terms $\delta \mathcal{L} \mathbf{u}_{0}$. The meaning of 'single scattering' is then that for each perturbed scattering point in the medium, we only consider scattering generated by the reference wavefield. This means that all energy in the scattered wavefield, $\delta \mathbf{u}$, has interacted with only one scattering point. Additionally, we neglect the effect of earlier scatterers on the reference wavefield as recorded at the receiver. Importantly, this second point means that the Born approximation does not conserve energy, because we assume that we can simply add in a small scattered wavefield and not change the reference wavefield. This predicts that the Born approximation will break down if the scattered wavefield becomes large relative to the reference wavefield.

There are many approaches in the literature describing techniques to go from the symbolic definition of the Born approximation in eq. (4) to practical techniques for modelling waveforms in a perturbed earth model. Until recently, most global applications of Born theory made assumptions to reduce both the types of scattering and integration volume, such as the path average approximation (PAVA; Woodhouse \& Dziewonski 1984), which reduces the sensitivity of the waveform to the average of the structure along the great-circle path, and non-linear asymptotic coupling theory (NACT; Li \& Romanowicz 1995), which includes coupling between mode branches to account for the heterogeneity of sensitivity along the great-circle path but uses a stationary phase approximation to ignore the contribution of sensitivity off the great-circle path, or the scalar 
exponent approximation (SEA; Marquering \& Snieder 1995), which uses approximate methods of treating surface-wave mode coupling to model along-path sensitivity of waveforms of body wave phases. In recent years, however, advances in computational power have led to practical means of computing fully 3-D Born sensitivity kernels for body waves using paraxial ray theory (Dahlen et al. 2000), for surface waves using surface wave modes (Meier et al. 1997; Zhou et al. 2004) and for long-period waveforms and delay times using normal modes (Capdeville 2005; Zhao \& Jordan 2006).

In this paper, we use the methodology described by Capdeville (2005), which efficiently considers full mode coupling, by using convolutions of mode wavefields at scattering points rather than explicit mode coupling analogous to the methods used in adjoint misfit gradient calculations (e.g. Tromp et al. 2005). With this formalism, we can define 3 -D sensitivity kernels, $\mathcal{K}(\mathbf{x}, t)$, as expressed by the integral relationship

$\delta \mathbf{u}(t)=\int_{V} \mathcal{K}(\mathbf{x}, t) \cdot \delta \mathbf{m}(\mathbf{x}) \mathrm{d} V$,

where the integration is over the volume of the Earth and $\delta \mathbf{m}$ is the model vector describing the perturbations to the elastic coefficients and density. An example kernel for shear velocity perturbations at a time point during a fundamental mode Rayleigh wave is shown in Fig. 1. Although minor differences exist between implementations of the first-order Born approximation, critical calculations were also validated using the method of Zhou et al. (2004), based on surface wave modes, which showed consistent results.

The classical normal mode based expression of the Born approximation is included as expression A1 in the Appendix. Direct implementation of this is computationally expensive as it requires an integration over the volume of the Earth for each possible pair of coupled modes. Additionally, the expression is non-linear for time domain waveforms. Indeed, as pointed out in Appendix as well as previous normal mode work (e.g. Tanimoto 1984; Romanowicz et al. 2008), this non-linearity is due to an approximation of incorporating a secular term that grows unbounded with time into the exponential of the equation. The term proportional to time is a short

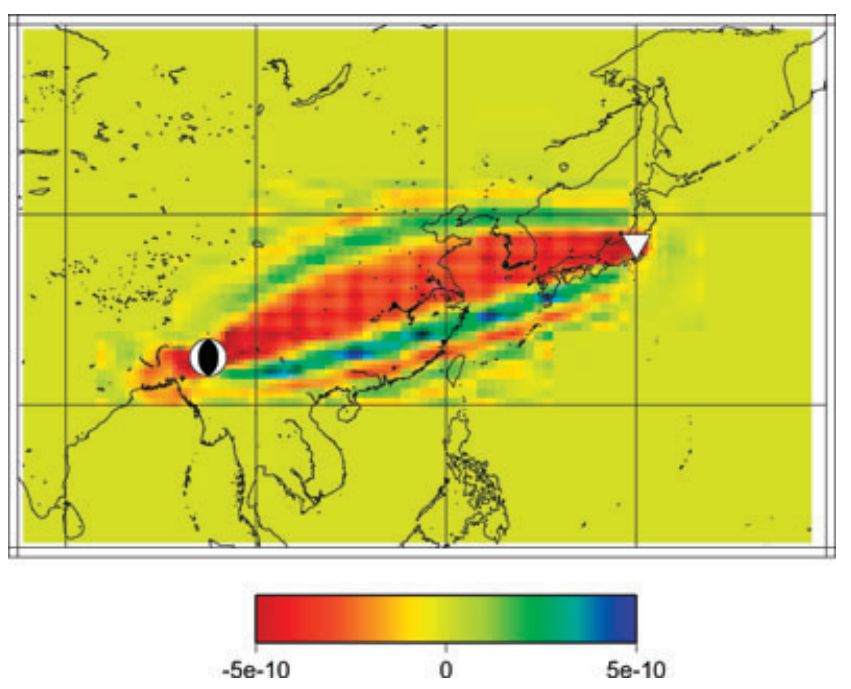

Figure 1. An example waveform sensitivity kernel at a depth of $65 \mathrm{~km}$ for a point in a fundamental mode Rayleigh wave packet recorded on the vertical component with minimum period of $60 \mathrm{~s}$. The white triangle is the receiver, and the source mechanism is shown at the source location. The scale shows the perturbation to the acceleration seismogram $\left(\mathrm{m} \mathrm{s}^{-2}\right)$ integrated over cells $1^{\circ} \times 1^{\circ}$ laterally and $100 \mathrm{~km}$ in depth. time approximation that arises when we derive the first-order Born approximation as a linear waveform perturbation. This secular term is a clear indicator of how the Born approximation breaks down if the model perturbation, and thus, the scattered wavefield and the secular term itself grows too large relative to the reference waveform. It is important to point out, however, if we instead derive expressions for linear frequency perturbations for modes (or equivalently phase delays in the frequency domain for surface waves or cross-correlation traveltime shifts for body waves), the same problem does not occur, as the secular term is not required. This difference points out why we would not necessarily expect the same range of validity for Born-based modelling of waveform perturbations versus modelling of phase delays, which we examine more fully in Section 6 .

All of current practical approaches for time-domain waveform modelling use techniques to deal with the two problems of computational expense and the treatment of the secular term. PAVA and NACT both use asymptotic approximations to spherical harmonics and the stationary phase approximations to reduce the integrations over the whole globe in eq. (A1) to integrations along the path between the source and receiver. Capdeville (2005) reduces computational cost by a reorganization of expression (A5) that avoids explicitly coupling every mode. Tanimoto (1984) suggests treating the secular term by absorbing it into the exponential, analogous to treating an expression of the form $(1+\delta \omega t) \exp (\mathrm{i} \omega t)$ as $\exp [\mathrm{i}(\omega+$ $\delta \omega) t]$. This basic idea of how to treat the secular term is also incorporated into PAVA and NACT, as discussed briefly in the Appendix and more fully in Romanowicz et al. (2008). However, current practical implementations of the Born approximation for modelling waveforms that fully account for 3-D structure (e.g. Capdeville 2005) do not include any correction to account for the unbounded growth of the secular term. This potential shortcoming is the primary motivation for this study to carefully test the validity of time domain waveform modelling using the first-order 3-D Born approximation.

\section{EVALUATING 3-D BORN WAVEFORM MODELLING WITH SIMPLE MODELS}

A critical point for evaluating seismic models derived using the Born approximation in time-domain waveform modelling is to understand how well the theory performs for forward modelling the effects of seismic structure. To systematically explore what situations we expect to gain accuracy using the 3-D Born approximation, and in what situations the assumptions of the approximation are violated, we set up a series of synthetic tests with simple source-receiver geometries and perturbation models.

We calculate numerical traces using a coupled normal-mode spectral element method code (CSEM, Capdeville et al. 2002; Chaljub et al. 2003). We treat the numerical traces as ground truth for evaluating predicted waveforms from 3-D Born kernels, as well as mode-based great-circle approximations PAVA (Woodhouse \& Dziewonski 1984) and NACT (Li \& Romanowicz 1995), which approximate the seismic sensitivity as an average of structure along the path, and as a 2-D function of sensitivity collapsed on the great-circle path, respectively. PAVA is discussed in more detail in Section 3.2 and NACT in the Appendix. Figs 2-5 also show traces labelled NBORN, which are calculated using an approach discussed in Section 4. All the seismic traces shown in Figs 2-5 are acceleration time-series that have been bandpass filtered with a cosine taper with cut-offs at 100 and $1000 \mathrm{~s}$ and corners at 120 and $250 \mathrm{~s}$ periods. The models shown all have maximum velocity anomalies near $220 \mathrm{~km}$ depth with the amplitude tapering 


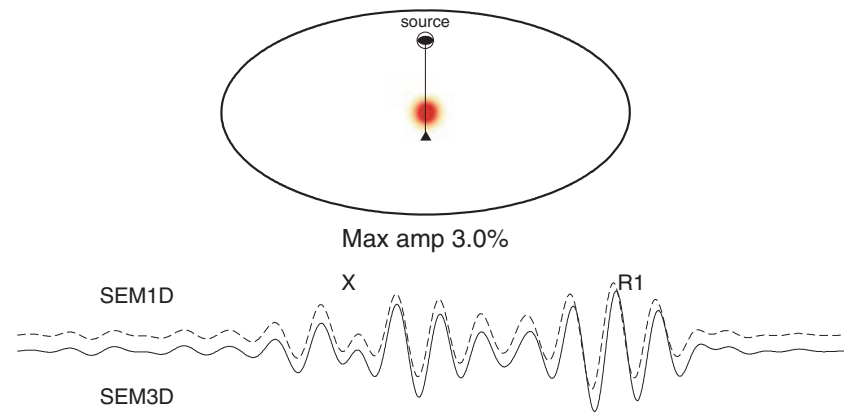

Differential traces X 2.55 (SEM differential dashed)
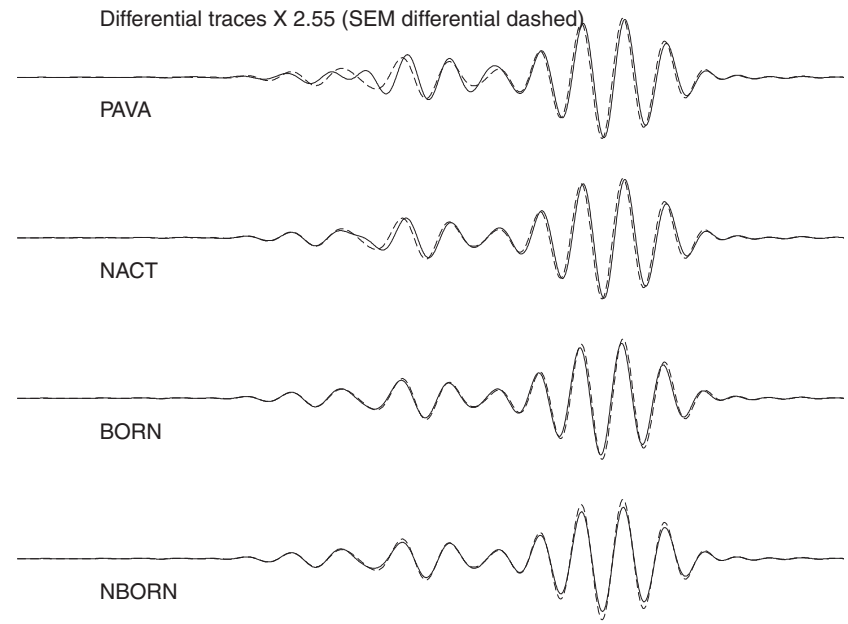

Misfit to SEM3D X 2.55

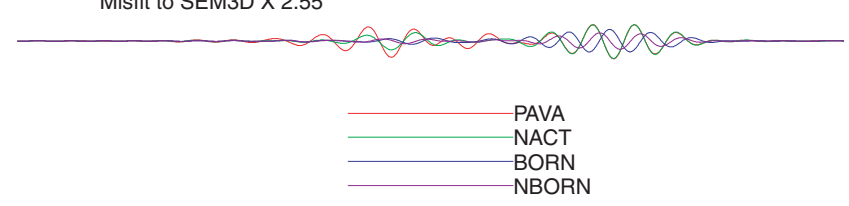

Figure 2. Comparisons of several theories to numerical synthetics. The model and path geometry is shown at top at a depth of $220 \mathrm{~km}$. The source is at a depth of $100 \mathrm{~km}$ and the receiver is at a distance of $80^{\circ}$ ( $\sim 8800 \mathrm{~km})$. The traces labelled SEM1D and SEM3D are numerical traces through the reference model and perturbed model, respectively, with a small vertical offset for clarity. Below are shown predicted differential traces (3-D predicted trace minus reference trace) in solid for PAVA, NACT, 3-D Born waveform kernels (BORN) and a modified Born trace (NBORN) overlaying the numerical differential trace as a dashed line. See Section 4 for a description of NBORN. The scale is magnified relative to the SEM1D and SEM3D traces by the factor shown above the PAVA trace. The bottom traces show the misfit for all methods relative to the numerical results at the same scale as the differential traces. See text in Section 3.1 for further description of the frequency band and model. $\mathrm{R}$ and $\mathrm{X}$ label the fundamental mode Rayleigh wave and overtone wave packets, respectively.

to zero at the surface and at $471 \mathrm{~km}$ depth. The radial amplitude of the structure is defined by a cubic b-spline (Mégnin \& Romanowicz 2000) with knots at depths of $0,121,221,321$ and $471 \mathrm{~km}$. The background velocity model for all calculations is a simplified version of PREM (Dziewonski \& Anderson 1981) with the crustal structure and the $220 \mathrm{~km}$ discontinuity removed.

\subsection{Examples where Born waveform modelling performs well}

As discussed by Spetzler et al. (2001), we expect that methods accurately taking into account 3-D sensitivity should outperform

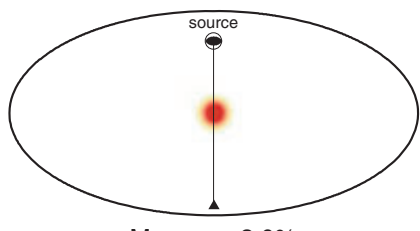

Max amp 3.0\%
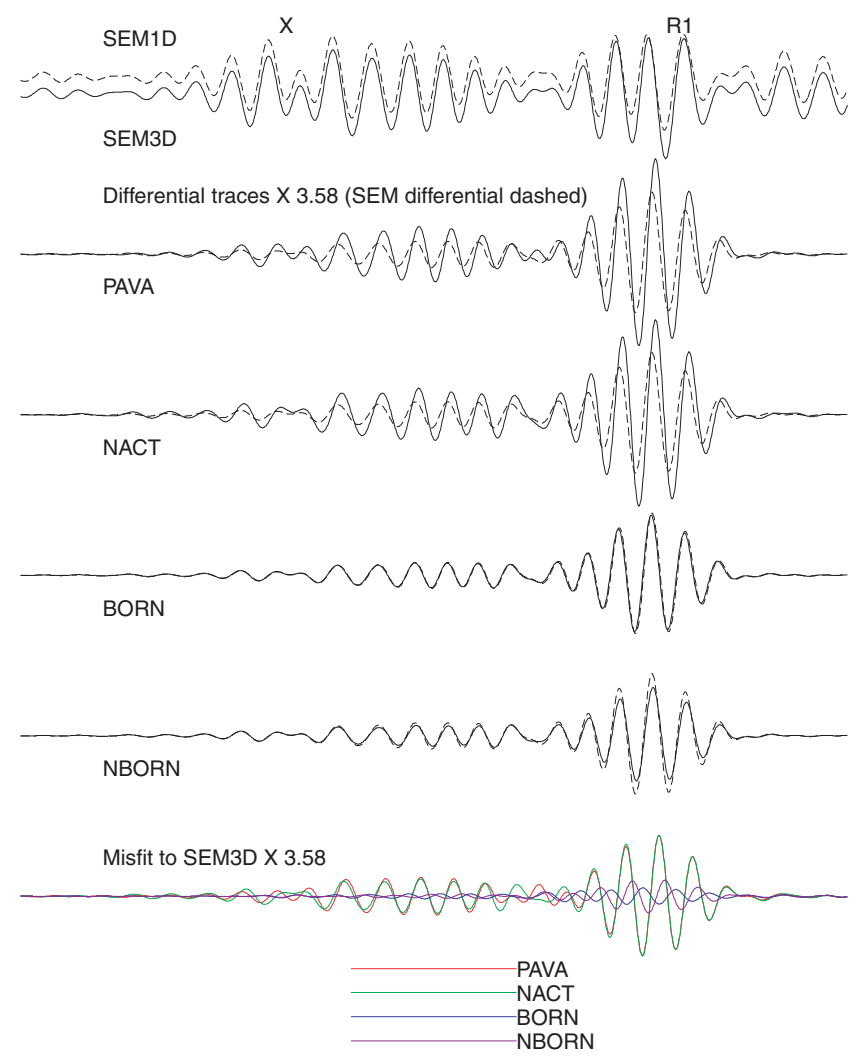

Figure 3. Same as Fig. 2 for the source receiver geometry shown at top, with an epicentral distance of $140^{\circ}(\sim 15500 \mathrm{~km})$.

great-circle based approximations when the size of the anomaly is similar to or smaller than the width of the Fresnel zone for the surface wave of interest. To verify this numerically, we define a model with lateral structure defined by a 2-D Gaussian function with a full width at half maximum of $12^{\circ}(\sim 1300 \mathrm{~km})$. For the particular background velocity model chosen here, this is comparable to an estimate of the width of the first Fresnel zone near the midpoint of the source-receiver path for the fundamental mode Rayleigh wave at $120 \mathrm{~s}$, the short-period corner of the passband.

When the source-receiver path goes directly through the anomaly and the receiver is located close to the anomaly (Fig. 2), the Fresnel zone is smaller than this anomaly, and the numerical trace is similarly well fit by PAVA, NACT and the first-order Born approximation. The last trace shown in Figs $2-5$, labelled NBORN, is explained in Section 4. For the fundamental mode (labelled R1), all theories do a good job of fitting the differential seismograms (defined by the predicted trace through the 3-D model minus the 1-D reference trace). This can be expected, as the Fresnel zone will be smaller than this anomaly near the source and receiver, and great-circle based approximations should be sufficient. In the portion of the seismogram with overtone surface wave energy, NACT and Born both show some improvement over PAVA. This shows that the heterogeneity of sensitivity along the path predicted by NACT for overtones when cross-branch coupling is included (Li \& 


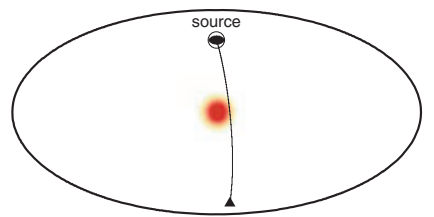

Max amp 3.0\%

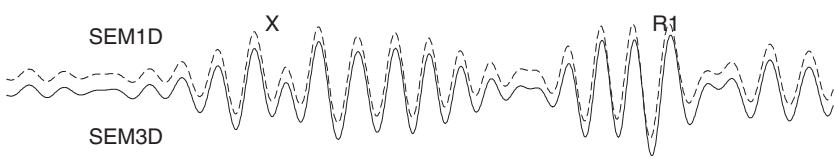

Differential traces X 4.19 (SEM differential dashed)
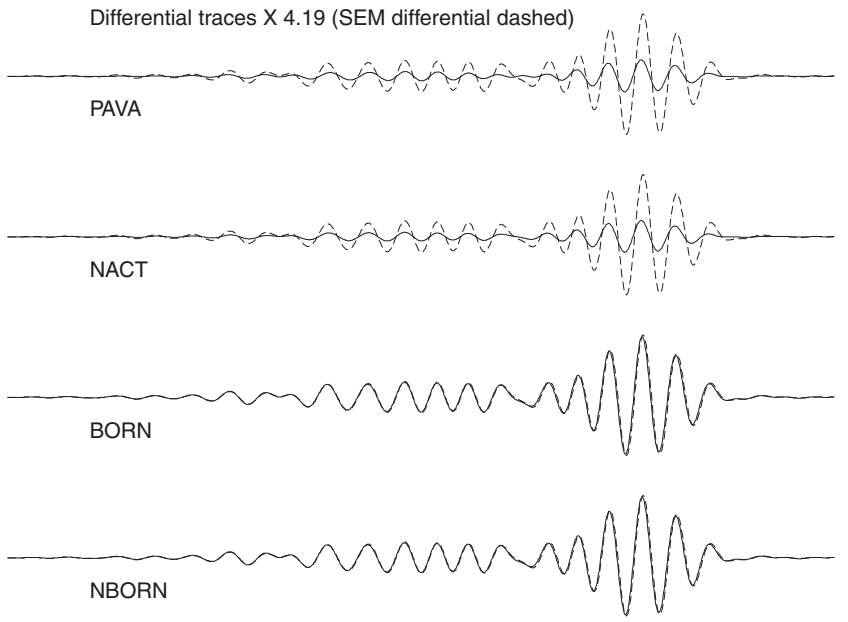

Misfit to SEM3D X 4.19

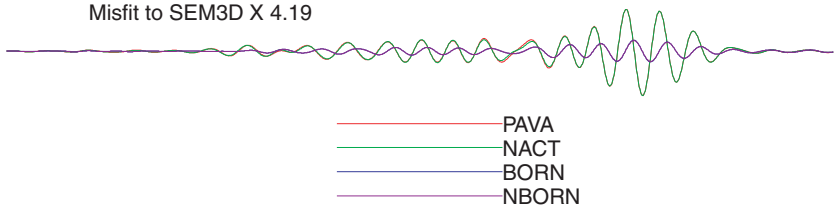

Figure 4. Same as Fig. 2 for the source receiver geometry shown at top with an epicentral distance of $140^{\circ}(\sim 15500 \mathrm{~km})$.

Romanowicz 1995) improves fit, even when PAVA does a good job for the fundamental mode. The first-order 3-D Born waveform kernels offer further modest improvement over NACT for the overtones in this case, meaning that the off-path distribution of the anomaly also plays a role in the predicted waveform here.

When the receiver is farther from the source, so that the anomaly is near the midpoint of the path (Fig. 3), accurately accounting for off-path sensitivity becomes more important. In this case, the whole anomaly is contained within the Fresnel zone, and both PAVA and NACT overpredict the effect of the anomaly. PAVA and NACT begin to show signs of failure because the methods assume that structure is relatively constant over the Fresnel zone, whereas in fact the anomaly here goes to zero near the edge of the predicted Fresnel zone, leading to the overprediction. Physically, one can also think of this as a wavefront healing effect (e.g. Hung et al. 2001) because paths through scatterers near the edge of the anomaly, which can still arrive constructively with energy propagating along the greatcircle path, do not see as much structure, thus lessening the total effect of the anomaly.

Similarly, we see that paths that graze the edge of the anomaly at this distance (Fig. 4), are also not correctly predicted by great-circle based approximations. In this case, PAVA and NACT underpredict the effect of the anomaly because the structure along the path is quite small in amplitude, whereas that immediately off the path but

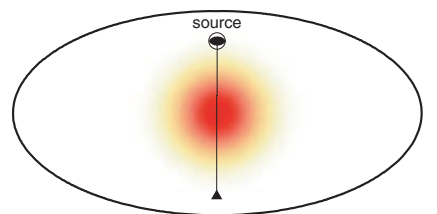

Max amp 6.5\%

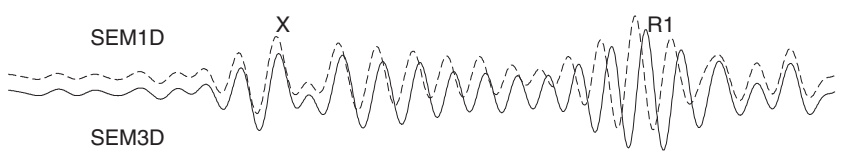

BM3
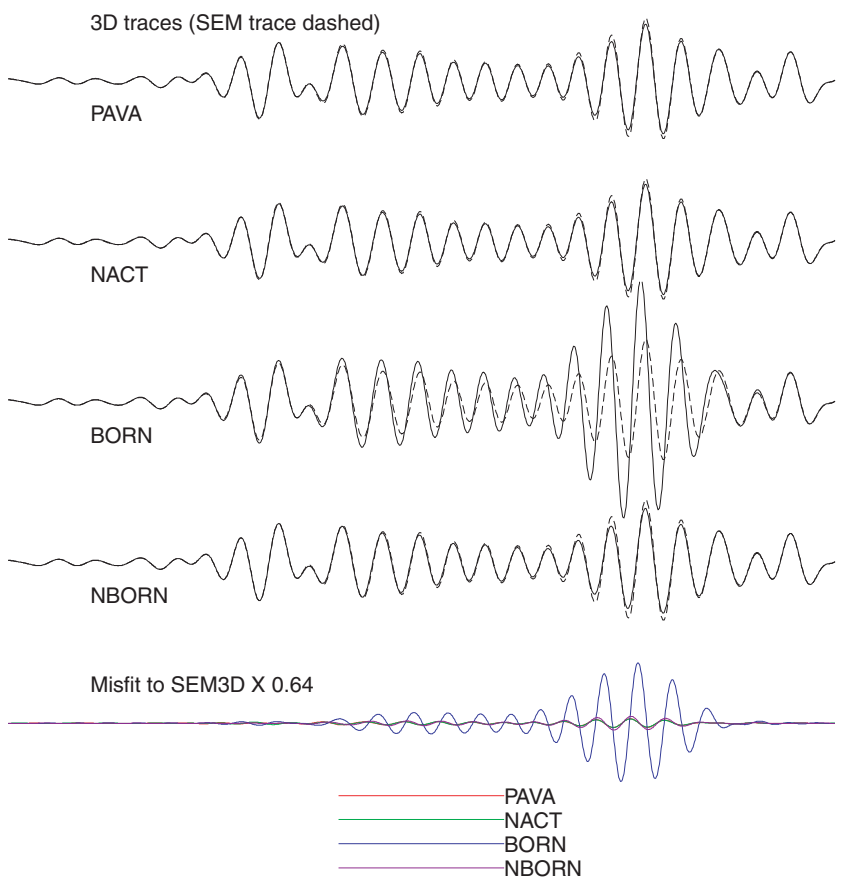

Figure 5. Same as Fig. 2 for the different model shown at top and for an epicentral distance of $130^{\circ}(\sim 14400 \mathrm{~km})$. See Section 3.2 for more details of the model. Traces labelled PAVA, NACT, BORN and NBORN are full 3-D traces plotted on the same scale as SEM1D and SEM3D rather than differential traces.

still contained within the Fresnel zone, is larger and still noteably affects the resulting seismogram.

\subsection{Example where first-order Born waveform modelling performs poorly}

Section 3.1 demonstrates the potential first-order Born waveform kernels present for improving resolution of shorter wavelength structure in global models. If great-circle based theories overpredict the effect of short wavelength structure, then models derived using these approaches may underpredict the amplitudes of such structure, producing artificially smooth models. However, it is also worthwhile to consider the predictions for long-wavelength structure, for which the great-circle based methods were designed. Do first-order Born kernels perform as well for this type of structure as the great circle methods?

To test this case, we assume a model with the same depth distribution as before, but the anomaly now has a full width at half maximum of $60^{\circ}$, which is much larger than the Fresnel zone for these surface waves. Additionally, the strength of the anomaly was increased from a peak of 3 per cent used in Section 3.1 to 
6.5 per cent. For a path directly through this large anomaly (Fig. 5), the relative performance of the different methods is quite different from before. For a large, high-amplitude anomaly, the effect on the seismogram should be much larger than in Section 3.1. When comparing the SEM1D reference trace to the SEM3D numerical trace, it is obvious that a large phase delay of a quarter cycle or more is introduced in the fundamental mode. Despite this large difference, the predicted seismograms (shown here as the full seismograms, rather than the differential seismograms) are well matched by both PAVA and NACT. Recalling the expression of the first-order Born approximation for time domain waveforms in Appendix, this would mean that the resulting secular term is larger and potentially more problematic. Indeed, the Born seismogram is more poorly matched in phase and significantly overpredicts the amplitude of the 3-D waveform, leading to a much greater misfit than for PAVA and NACT.

The breakdown of the first-order Born approximation in this case can be explained by the growth of the secular term, which is a function of the assumptions inherent in the method. As discussed in Section 2, the first-order Born approximation defined in eq. (4) does not conserve energy. When the scattered wavefield is comparable to the amplitude of the reference wavefield, it is no longer appropriate to neglect the higher order terms in eq. (4). For $\delta \mathbf{u}$ to be large enough to produce an apparent phase shift of more than a quarter cycle, it is clear that it must have an amplitude similar to the reference trace, and thus the Born approximation breaks down. Mathematically, this is expressed by the unbounded growth of the secular term. From the physical point of view of neglecting energy conservation, we can see that we are adding energy into the wavefield beyond that supplied by the source, and thus we greatly overpredict the amplitude of the resulting waveform.

PAVA and NACT do not suffer the same breakdown because they incorporate this secular term as a linear phase perturbation inside the exponential rather than a linear waveform perturbation. This sort of expression is valid at long times, and, as discussed below, also includes some contribution from multiple scattering along the great circle (e.g. Friederich et al. 1993, appendix).

It is worthwhile to consider in more detail how this phase perturbation is implemented in PAVA, and why this leads to the better fit for model perturbations such as that shown in Fig. 5. The reference 1-D seismogram can be written as a sum of normal modes

$\mathbf{u}_{0}(t)=\sum_{k=0}^{N} \mathbf{u}_{k}(t)$,

where $N$ is the number of normal modes in the frequency band of interest, and $\mathbf{u}_{k}$ is defined as

$\mathbf{u}_{k}(\Delta, t)=\mathbf{A}_{k}(\Delta) \cos \left(\omega_{k} t\right)$

where $\Delta$ is the epicentral distance, $\omega_{k}$ is the eigenfrequency of the mode in the reference model and $\mathbf{A}_{k}$ is an excitation and phase term related to the eigenfunctions at the source and receiver depths and generalized associated Legendre functions (Phinney \& Burridge 1973) of the epicentral distance (see eq. A11).

The PAVA approximation, as introduced by Woodhouse \& Dziewonski (1984) for waveform modelling, is expressed as

$\mathbf{u}_{\mathrm{PAVA}}(t)=\sum_{k} \mathbf{A}_{k}(\Delta+\delta \Delta) \cos \left(\hat{\omega}_{k} t\right)$,

where

$\hat{\omega}_{k}=\omega_{k}+\delta \hat{\omega}$, $\delta \Delta=\frac{\Delta}{U_{k}\left(l+\frac{1}{2}\right)}\left(\delta \hat{\omega}-\delta \tilde{\omega}_{k}\right)$

$\delta \hat{\omega}_{k}=\frac{1}{2 \pi} \int_{0}^{2 \pi} \delta \omega_{\text {local }}\left(\phi^{\prime}\right) \mathrm{d} \phi^{\prime}$

$\delta \tilde{\omega}_{k}=\frac{1}{\Delta} \int_{0}^{\Delta} \delta \omega_{\text {local }}\left(\phi^{\prime}\right) d \phi^{\prime}$

where $U_{k}$ is group velocity of the $k$ th mode, $\delta \omega_{\text {local }}$ is the local frequency as defined by Jordan (1978), $\delta \hat{\omega}_{k}$ is the great-circle average of the frequency shift and $\delta \tilde{\omega}_{k}$ is the minor arc average. The variable $\phi^{\prime}$ refers to the set of coordinates $(\theta, \phi)$ along the great-circle path defined by the source and receiver. This expression was initially proposed as an ad hoc approach to waveform modelling (Woodhouse \& Dziewonski 1984) but was later shown (Romanowicz 1987) to be equivalent to incorporating the secular term as a frequency shift in the exponential, as proposed by Tanimoto (1984), but only evaluating the contribution from coupling between modes along a single dispersion branch and collapsing the integrals to the great-circle path using asymptotic expansions of spherical harmonics and the stationary phase approximation.

The combination of frequency shift and apparent epicentral distance shift produces the appropriate phase shift with sensitivity limited to the propagation path for both minor and major arc, as well as multiple orbit phases. Conveniently for practical implementation, there is no need to track the orbit number of the phase, and wave packets containing both fundamental and overtone energy that may be from different orbit numbers are handled correctly. Because the structural effect is implemented as a phase shift rather than a simple addition of a scattered wavefield $\delta \mathbf{u}$, the energy conservation problem is partially avoided as a linear phase shift simply displaces energy in time in a wavefield. Although not a rigorous proof, one can intuitively think about this by comparing a linear waveform perturbation definition to a sine wave, $u=\sin t+\delta \phi \cos t$, to the equivalent linear phase perturbation, $u=\sin (t+\delta \phi)$. As the perturbation $\delta \phi$ goes to infinity, the total energy in the wave, $\int_{-\infty}^{\infty} u^{2} \mathrm{~d} t$, grows unbounded for the waveform perturbation while remaining unchanged for the phase perturbation. Romanowicz (1987) showed that the PAVA expressions are equivalent to standard measurements of phase velocities of surface waves for long-wavelength structure, and the numerical results shown in Fig. 5 convincingly show this to be the case. This excellent data fit can be further understood by considering that the PAVA solution approximates the contribution of multiple forward scattering to the perturbed wavefield. As has been shown by studying multiple scattering of surface waves (Friederich et al. 1993, appendix), the contribution of the multiply scattered wavefield (including all forward scattering, but neglecting backscattering and surface wave mode coupling) for a band heterogeneity of length $a$ along the path and infinite extent perpendicular to the path with a constant perturbation to the local wavenumber, $\Delta k$, is a simple phase shift $a \Delta k$. Given that result, the PAVA expression then correctly includes multiple forward scattering within the approximation that anomalies that vary smoothly along the path are well modelled by a path-averaged anomaly, and that the lateral extent of the anomalies are greater than the width of the Fresnel zone. NACT also does an equally good job in this case as it is implemented as a PAVA expression with additional asymptotic terms related to coupling between modes across dispersion branches.

It is important to note that this breakdown of the linear Born approximation results whenever the accumulated phase delay in 
radians is great enough. The particular model used to demonstrate this problem in Fig. 5 may be somewhat unrealistic, but is chosen to demonstrate the effect for the very long-period data shown here (dominant period greater than $120 \mathrm{~s}$ ) for a relatively short path. However, for shorter periods and/or longer paths, it is quite reasonable to obtain phase delays of greater than $\pi / 2$ for more modest velocity anomalies. For example, a mean velocity perturbation of only 0.5 per cent is sufficient to get such a delay for a minor arc $60 \mathrm{~s}$ surface wave propagating a distance of $120^{\circ}$. As a rule of thumb, the first-order Born prediction starts to noteably diverge from numerical predictions when the accumulated phase delay exceeds a quarter period. Given that, a very rough estimate to determine whether the linear Born approximation will begin to breakdown for prediction of surface waveforms at a desired frequency through a given model can then be obtained by considering whether the quantity

$\left\langle\frac{\delta c}{c}\right\rangle \omega t>\frac{\pi}{2}$

where $\langle\delta c / c\rangle$ is the path-averaged phase velocity perturbation at frequency $\omega$ and $t$ is the arrival time of the particular surface wave mode considered. This demonstrates why more modest velocity anomalies than that shown in the simple example above may be sufficient to violate the assumptions of the first-order Born approximation for longer paths and/or higher frequencies.

\section{MODIFICATION TOBORN WAVEFORM MODELLING}

For the simple models described above, it is clear that modelling based upon the linear 3-D Born approximation is preferable for some geometries but performs poorly in others. This is not an ideal case for implementation in a tomographic inversion. It is not generally feasible to pick and choose which approach to use for each seismic trace in the database, and clearly many real source-receiver geometries in the real Earth will incorporate elements of more than one of the simple cases demonstrated above. We would desire to have a single expression for modelling the waveforms that performs well for the geometries described in both Sections 3.1 and 3.2.

We look to define such an expression in a similar fashion as the derivation of NACT ( $\mathrm{Li} \&$ Romanowicz 1995). In the Appendix, we discuss how to apply the first-order Born approximation in a normal mode formalism, and briefly describe how such an approach is modified to extend the range of validity, as in NACT. Eq. (A9), which is the Born approximation linearized with respect to a set of modes with frequencies modified as predicted by path-averaged structure rather than the frequencies in the initial 1-D model, would be an excellent candidate for our desired expression that acts as a compromise between PAVA and first-order 3-D Born theory. It effectively linearizes the problem using modes that better reflect the 1-D structure between source and receiver, analogous to a method suggested by Meier et al. (1997), which advocated using non-linear inversion to use the best 1-D model for each path before applying first-order Born kernels. The expression gains longer time stability by using the higher order terms from a PAVA phase shift, but replacing its linear contribution with that accurately calculated from considering all mode coupling in the volume. There are, however, significant computational hurdles to using this equation directly in a model inversion. Because efficient implementations that consider full 3-D integration (e.g. Capdeville 2005) do not explicitly consider individual mode coupling, expression (A9) cannot be implemented directly. Additionally, we would require an expensive recomputation of the 3-D Born kernels for each path after each model iteration, as the definition of $\delta \tilde{\omega}$ depends on the previous model iteration. Furthermore, implementation of PAVA as in (A9), using only frequency shifts requires careful orbit tracking to determine precisely the path over which to integrate $\delta \omega_{\text {local }}$, which becomes complicated when waveforms include overlapping energy from fundamental modes and higher modes from different orbits. This is not required when the apparent epicentral shift is used as in eq. (8).

To balance these computational concerns, we propose an approach that incorporates the PAVA phase shift in the exponential but restores the original Born sum of eq. (A4), as

$\mathbf{u}(t)=\mathbf{u}_{\mathrm{PAVA}}(t)+\delta \mathbf{u}_{\mathrm{Born}}(t)-\delta \mathbf{u}_{\mathrm{PAVA}}(t)$,

where $\mathbf{u}_{\text {PAVA }}$ is defined as in eq. (8), and $\delta \mathbf{u}_{\text {Born }}$ is defined as in eq. (A5). We want an expression $\delta \mathbf{u}_{\mathrm{PAVA}}$, so that we can write the linear expression

$\mathbf{u}_{\mathrm{PAVA}}(t) \approx \mathbf{u}_{0}(t)+\delta \mathbf{u}_{\mathrm{PAVA}}(t)$,

correct for short times and small values of $\delta \omega$. Such an expression can be defined by extracting two terms, $\delta \mathbf{u}_{\mathrm{PAVA}}=\delta \mathbf{u}_{\mathrm{PAVA}}^{(1)}+$ $\delta \mathbf{u}_{\mathrm{PAVA}}^{(2)}$, which we compute asymptotically to order zero, as derived by Romanowicz (1987); See also Romanowicz et al. (2008):

$\delta \mathbf{u}_{\mathrm{PAVA}}^{(1)}=\operatorname{Re}\left[\sum_{k} \mathbf{A}_{k} \delta \hat{\omega}_{k} \mathrm{i} t \exp \left(\mathrm{i} \omega_{k} t\right)\right]$,
$\delta \mathbf{u}_{\mathrm{PAVA}}^{(2)}=\operatorname{Re}\left[\sum_{k} \delta \Delta \mathbf{A}_{k}^{\prime}(\Delta) \exp \left(\mathrm{i} \omega_{k} t\right)\right]$,

where $\delta \Delta$ and $\delta \hat{\omega}_{k}$ are defined as in eqs (10) and (11), respectively, and $\mathbf{A}_{k}^{\prime}=\mathrm{d}_{k} / \mathrm{d} \Delta$ is the derivative of the excitation terms with respect to $\Delta$ (not derived here). $\delta \mathbf{u}_{\mathrm{PAVA}}^{(1)}$ arises from self-coupling (i.e. coupling of modes within a multiplet), whereas $\delta \mathbf{u}_{\mathrm{PAVA}}^{(2)}$ arises from consideration of coupling of modes along a dispersion branch. Adding these terms to $\mathbf{u}_{0}$ gives us the desired expression

$$
\begin{aligned}
\mathbf{u}_{\mathrm{PAVA}} \approx & \operatorname{Re}\left\{\sum_{k}\left[\mathbf{A}_{k}(\Delta)+\mathbf{A}_{k}(\Delta) \delta \hat{\omega}_{k} \mathrm{i} t+\delta \Delta \mathbf{A}_{k}^{\prime}(\Delta)\right] \times \exp \left(\mathrm{i} \omega_{k} t\right)\right\} \\
\approx & \mathbf{A}_{k}(\Delta) \cos \left(\omega_{k} t\right)+\delta \Delta \mathbf{A}_{k}^{\prime}(\Delta) \cos \left(\omega_{k} t\right) \\
& -t \delta \hat{\omega}_{k} \mathbf{A}_{k}(\Delta) \sin \left(\omega_{k} t\right) .
\end{aligned}
$$

Substituting the definitions of $\mathbf{u}_{\mathrm{PAVA}}$ and $\delta \mathbf{u}_{\mathrm{PAVA}}$ into eq. (15), we obtain

$$
\begin{aligned}
\mathbf{u}(t)= & \sum_{k}\left[\mathbf{A}_{k}(\Delta+\delta \Delta) \cos \left(\hat{\omega}_{k} t\right)-\delta \Delta \mathbf{A}_{k}^{\prime}(\Delta) \cos \left(\omega_{k} t\right)\right. \\
& \left.+t \delta \hat{\omega}_{k} \mathbf{A}_{k}(\Delta) \sin \left(\omega_{k} t\right)\right]+\delta \mathbf{u}_{\mathrm{Born}}(t) .
\end{aligned}
$$

This expression is correct to the same level of approximation in $\delta \omega$ as the original Born expression for time domain waveforms.

Intuitively, we can think of eq. (18) as simply taking the PAVA expression and removing the linear portions of the PAVA phase shift and replacing them with those predicted from the 3-D Born approximation. The two terms of $\delta \mathbf{u}_{\mathrm{PAVA}}$ can then be considered equivalently as the linear terms arising from a Taylor expansion of the PAVA expression around the reference frequency and epicentral distance. We can express the linearized version of PAVA from eq. (8) by taking the partial derivatives of eq. (7) with respect to $\Delta$ and $\omega$,

$$
\begin{aligned}
\frac{\partial}{\partial \Delta} \mathbf{u}_{k} & =\mathbf{A}_{k}^{\prime}(\Delta) \cos \left(\omega_{k} t\right), \\
\frac{\partial}{\partial \omega} \mathbf{u}_{k} & =-t \mathbf{A}_{k}(\Delta) \sin \left(\omega_{k} t\right) .
\end{aligned}
$$


We can use these to expand PAVA in a Taylor series around the reference frequency and epicentral distance to remove the linearized contribution of the frequency shift and apparent epicentral distance shift. We then obtain

$$
\begin{aligned}
\mathbf{u}_{k}(\Delta, t)= & {\left[\mathbf{A}_{k}(\Delta)+\delta \Delta \mathbf{A}_{k}^{\prime}(\Delta)+\mathcal{O}\left(\delta \Delta^{2}\right)\right] } \\
& \times\left[\cos \left(\omega_{k} t\right)-t \delta \hat{\omega}_{k} \sin \left(\omega_{k} t\right)+\mathcal{O}\left(\delta \hat{\omega}_{k}^{2}\right)\right] \\
\approx & \mathbf{A}_{k}(\Delta) \cos \left(\omega_{k} t\right)+\delta \Delta \mathbf{A}_{k}^{\prime}(\Delta) \cos \left(\omega_{k} t\right) \\
& -t \delta \hat{\omega}_{k} \mathbf{A}_{k}(\Delta) \sin \left(\omega_{k} t\right),
\end{aligned}
$$

which is equivalent to eq. (17).

If we then remove the linearized PAVA contribution from the full linear mode coupling term, $\delta \mathbf{u}_{\text {Born }}$, we would once again obtain eq. (18), which shows that equation can be considered as correcting the linear terms of the expression for $\mathbf{u}_{\text {PAVA }}$ with the more accurate 3-D Born terms. Note that if the path-averaged model perturbations are small enough so that the PAVA phase shift is small, and the waveform perturbations are well modelled by just the linear terms, $\mathbf{u}_{0}-\delta \mathbf{u}_{\text {PAVA }}$ is simply the reference trace, $\mathbf{u}_{0}$, and we have the simple first-order Born approximation. However, if the PAVA phase delay is large, the higher order terms become more important and act as a correction to the first-order Born expression.

Eq. (18) does a remarkable job for the simple cases discussed in Sections 3.1 and 3.2, with negligible additional computation with respect to the standard first-order 3-D Born kernels. As can be seen in the traces labelled NBORN in Figs 2-5, the misfit for this approach is comparable to that of whichever method had performed best. Although there may be a slight increase in misfit relative to the best trace in each case, it clearly avoids both the breakdowns that plague the great-circle approximation for small anomalies, as well as those that affect the Born approximation for large phase delays.

Of course, the ultimate goal of these expressions is to improve the resulting tomographic models. The modified Born approximation derived here changes the inversion process in two ways. As shown for simple models in Sections 3.1 and 3.2 and for more complicated models in the following Section 5, it improves the forward modelling. This is an important component for iterative inversions when starting from a non-zero model. Second, the partial derivatives with respect to model parameters that are non-zero along the great-circle path will be modified based on the non-zero starting model.

We'll consider a model parametrized as

$\delta \mathbf{m}(r, \theta, \phi)=\sum_{p} \mathbf{c}_{p} h_{p}(r, \theta, \phi)$,

where $\mathbf{m}$ and $\mathbf{c}_{p}$ are vectors to allow for multiple physical parameters (e.g. $V_{P}, V_{S}$, and $\rho$ ) and $h_{p}(r, \theta, \phi)$ represents the set of spatial basis functions. Then we can write the partial derivatives based on expression (18) as

$\frac{\partial \mathbf{u}}{\partial \mathbf{c}_{p}}=\frac{\partial \mathbf{u}_{1}}{\partial \mathbf{c}_{p}}+\mathcal{K}_{p}^{(\text {Born })}$

where $\mathcal{K}_{p}^{(\text {Born })}$ is the Born kernel as calculated by Capdeville (2005), integrated over the basis function $h_{p}(r, \theta, \phi)$. Sensitivity to model structure enters $\mathbf{u}_{1}$ through $\delta \hat{\omega}$ and $\delta \tilde{\omega}$, as defined in eqs (11) and (12). Using those expressions and eq. (A3), we can then write

$\frac{\partial \mathbf{u}_{1}}{\partial \mathbf{c}_{p}}=\frac{\partial \mathbf{u}_{1}}{\partial \delta \hat{\omega}} \frac{\partial \delta \hat{\omega}}{\partial \mathbf{c}_{p}}+\frac{\partial \mathbf{u}_{1}}{\partial \delta \tilde{\omega}} \frac{\partial \delta \tilde{\omega}}{\partial \mathbf{c}_{p}}$

$$
\begin{aligned}
= & \frac{\Delta}{U_{k}\left(l+\frac{1}{2}\right)}\left[\mathbf{A}_{k}^{\prime}(\Delta+\delta \Delta) \cos \left(\hat{\omega}_{k} t\right)-\mathbf{A}_{k}^{\prime}(\Delta) \cos \left(\omega_{k} t\right)\right] \\
& \times\left(\frac{\partial \delta \hat{\omega}}{\partial \mathbf{c}_{p}}-\frac{\partial \delta \tilde{\omega}}{\partial \mathbf{c}_{p}}\right) \\
& -t\left[\mathbf{A}_{k}(\Delta+\delta \Delta) \sin \left(\hat{\omega}_{k} t\right)-\mathbf{A}_{k}(\Delta) \sin \left(\omega_{k} t\right)\right] \frac{\partial \delta \hat{\omega}}{\partial \mathbf{c}_{p}}
\end{aligned}
$$

where

$\frac{\partial \delta \hat{\omega}}{\partial \mathbf{c}_{p}}=\frac{1}{2 \pi} \int_{0}^{2 \pi} \int_{0}^{a} \mathbf{M}_{k k}(r) h_{p}\left(r, \phi^{\prime}\right) \mathrm{d} r \mathrm{~d} \phi^{\prime}$,

$\frac{\partial \delta \tilde{\omega}}{\partial \mathbf{c}_{p}}=\frac{1}{\Delta} \int_{0}^{\Delta} \int_{0}^{a} \mathbf{M}_{k k}(r) h_{p}\left(r, \phi^{\prime}\right) \mathrm{d} r \mathrm{~d} \phi^{\prime}$

and $\mathbf{M}_{k k}(r)$ are the radial sensitivity kernels used in eq. (A3).

The expressions in square brackets in eq. (25) demonstrate that this term is a modification to the Born partial derivatives that goes to zero unless there is a non-zero starting model (i.e. $\delta \Delta, \delta \hat{\omega}$ and $\delta \tilde{\omega}$ are non-zero). Eq. (27) shows that this modification is applied only for model parameters with a spatial basis function that is non-zero along the great-circle path between source and receiver.

This modification of the partial derivatives based on the starting model means that the inversion is non-linear in the sense that the partial derivatives depend on the starting model. However, the underlying calculation of the eigenfunctions, used to define both the Born kernels and the PAVA modifications to the forward calculation, and partial derivatives is based on the original 1-D reference model. Although this is not the same as a fully non-linear inversion with all derivatives calculated from a 3-D starting model, it allows for approximate corrections to the forward calculation and derivatives for the starting 3-D structure without the computational expense of numerical approaches.

\section{EVALUATION OF PERFORMANCE IN EARTH-LIKE MODELS}

While evaluating performance in simple models is informative for determining specific strengths and weaknesses of different methods, it is not clear how to extrapolate such results to realistic earth models. To truly evaluate the forward calculation performance of these methods, we would want to compare the misfits for many paths through the real Earth to determine which method performs the best in a statistical sense. However, the structure of the real Earth is not known, and simulating many paths for many events in these different methods can become computationally expensive. To get some sort of measure of the methods in realistic earth models in a computationally efficient manner, we simulated paths with multiple distances and azimuths from a single event in several random 'Earthlike' models. We choose to use several random models rather than any specific existing tomographic model to allow us to simulate many paths from events in different portions of the Earth, using a single event location in the calculation of the numerical traces and theoretical predictions. Otherwise our results could be biased by strong structure near the repeated source.

Of course, we do not know precisely what the Earth looks like, and so, we have to make a decision as to what the characteristics are that define an 'Earth-like' model. We choose to define random models that have shear velocity structure characteristic wavelengths and amplitudes similar to that of the global model SAW24B16 (Mégnin \& Romanowicz 2000). We generated three random upper-mantle velocity models with spherical harmonic power spectra and radial 
correlation fixed to that of SAW24B16 (Mégnin \& Romanowicz 2000, figs 6 and 15), with the exception of the degree 0 (spherically symmetric) term, which is set to zero for the random models. For the long-wavelength structure, this model is similar spectrally to other global models and is a reasonable estimate of the type of spherical harmonic power expected in long-wavelength structure. We then calculated seismograms through the second orbit fundamental mode Rayleigh wave at six different azimuths at four distances (e.g. Fig. 6a). However, SAW24B16, like other global models, includes smoothing that will tend to bias shorter wavelength structure to zero. For this reason, we made another set of three models with the same spectral power as SAW24B16 to degree 12 but with a white spectrum extended out to degree 24 with power set to the mean power in degrees 8-12 in SAW24B16. This class of models appears somewhat more spotty and has somewhat higher overall amplitudes than the SAW24B16-like models (e.g. Fig. 6b).

For all models, we calculated 24 vertical component seismograms to $10000 \mathrm{~s}$. These were bandpass filtered with cut-off frequencies at 80 and $1000 \mathrm{~s}$, and corners at 100 and $250 \mathrm{~s}$ period. These were calculated with CSEM, as well as PAVA, NACT, Born kernels and the modified Born approximation. With this data, we can systematically compare misfits as a function of distance and methodology, treating the CSEM synthetics as ground truth. We calculated misfits for each trace for windows centered on the first and second orbit
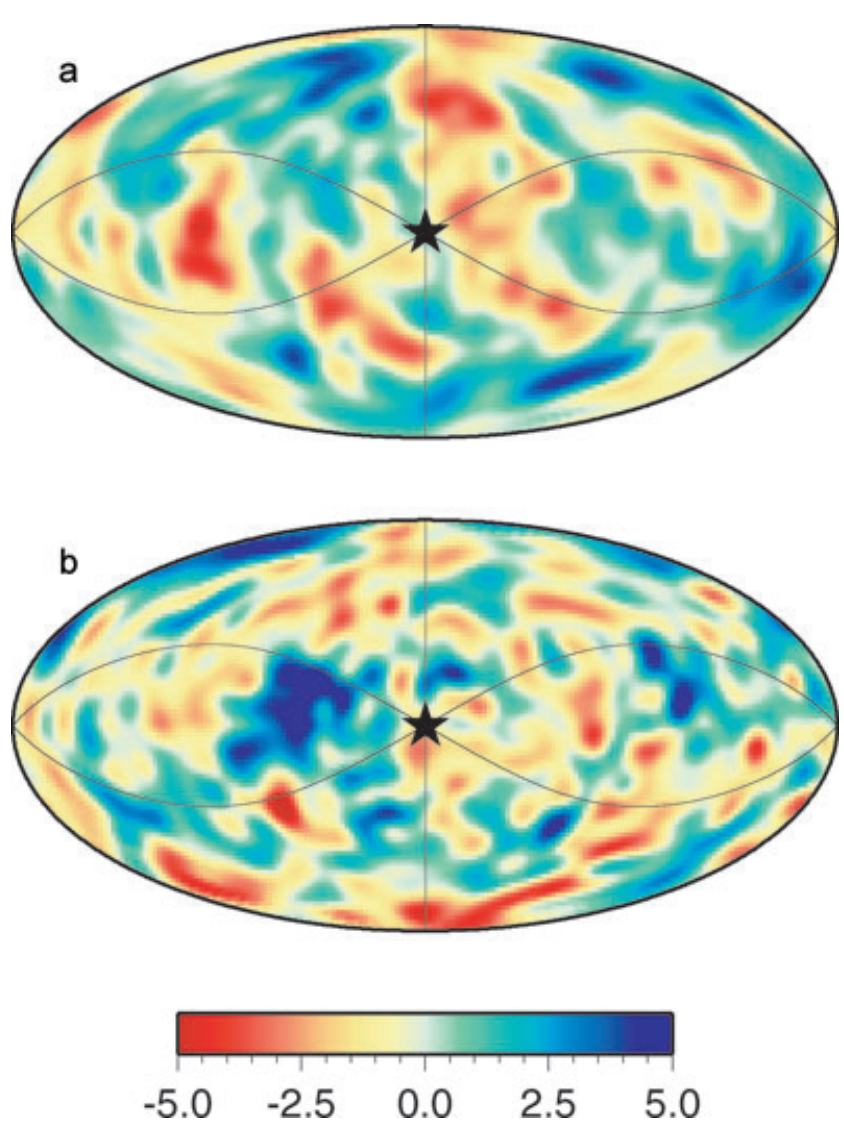

Figure 6. Example slices at a depth of $200 \mathrm{~km}$ for (a) a random model with the same wavelength characteristics of SAW24B16 and (b) a random model with a whitened spectrum at spherical harmonic degree 13-24. The source location used in the simulations is shown with a star, and the six azimuths on which traces were calculated are shown as solid lines. The colour scale shows $S$ velocity perturbation (per cent).
Table 1. RMS misfit normalized by rms amplitude of 3-D SEM trace.

\begin{tabular}{|c|c|c|c|c|c|c|c|c|c|}
\hline \multirow[b]{2}{*}{ Distance } & \multirow[b]{2}{*}{ Models } & \multicolumn{2}{|c|}{ PAVA } & \multicolumn{2}{|c|}{ NACT } & \multicolumn{2}{|c|}{ BORN } & \multicolumn{2}{|c|}{ NBORN } \\
\hline & & $\mathrm{R}$ & $X$ & $\mathrm{R}$ & $X$ & $\mathrm{R}$ & $\mathrm{X}$ & $\mathrm{R}$ & $\mathrm{X}$ \\
\hline \multirow{3}{*}{20} & All & 0.14 & 0.15 & 0.14 & 0.14 & 0.28 & 0.27 & 0.21 & 0.22 \\
\hline & SAW & 0.14 & 0.15 & 0.14 & 0.14 & 0.26 & 0.27 & 0.21 & 0.21 \\
\hline & Whitened & 0.14 & 0.15 & 0.14 & 0.14 & 0.29 & 0.27 & 0.22 & 0.22 \\
\hline \multirow{3}{*}{60} & All & 0.17 & 0.19 & 0.16 & 0.15 & 0.74 & 0.36 & 0.26 & 0.17 \\
\hline & SAW & 0.17 & 0.19 & 0.16 & 0.14 & 0.82 & 0.40 & 0.27 & 0.18 \\
\hline & Whitened & 0.17 & 0.18 & 0.16 & 0.15 & 0.64 & 0.32 & 0.26 & 0.17 \\
\hline \multirow{3}{*}{100} & All & 0.29 & 0.26 & 0.29 & 0.22 & 1.10 & 0.41 & 0.42 & 0.23 \\
\hline & SAW & 0.26 & 0.25 & 0.26 & 0.21 & 1.21 & 0.46 & 0.43 & 0.23 \\
\hline & Whitened & 0.32 & 0.27 & 0.32 & 0.24 & 1.00 & 0.37 & 0.41 & 0.23 \\
\hline \multirow{3}{*}{150} & All & 0.67 & 0.38 & 0.66 & 0.40 & 1.69 & 0.41 & 1.00 & 0.31 \\
\hline & SAW & 0.56 & 0.35 & 0.55 & 0.33 & 1.57 & 0.35 & 0.85 & 0.24 \\
\hline & Whitened & 0.78 & 0.42 & 0.78 & 0.46 & 1.82 & 0.47 & 1.15 & 0.38 \\
\hline \multirow{3}{*}{210} & All & 0.87 & 0.62 & 0.87 & 0.61 & 2.17 & 0.85 & 1.06 & 0.58 \\
\hline & SAW & 0.75 & 0.55 & 0.75 & 0.53 & 2.27 & 0.82 & 1.09 & 0.54 \\
\hline & Whitened & 1.00 & 0.69 & 1.00 & 0.70 & 2.07 & 0.88 & 1.03 & 0.63 \\
\hline \multirow{3}{*}{260} & All & 0.55 & 0.42 & 0.55 & 0.39 & 1.69 & 0.57 & 0.60 & 0.38 \\
\hline & SAW & 0.43 & 0.38 & 0.42 & 0.34 & 1.82 & 0.54 & 0.56 & 0.34 \\
\hline & Whitened & 0.68 & 0.46 & 0.68 & 0.44 & 1.55 & 0.60 & 0.64 & 0.43 \\
\hline \multirow{3}{*}{300} & All & 0.60 & 0.44 & 0.59 & 0.42 & 1.70 & 0.48 & 0.75 & 0.39 \\
\hline & SAW & 0.50 & 0.40 & 0.49 & 0.33 & 1.40 & 0.39 & 0.63 & 0.30 \\
\hline & Whitened & 0.70 & 0.49 & 0.70 & 0.50 & 2.00 & 0.58 & 0.87 & 0.47 \\
\hline \multirow{3}{*}{340} & All & 0.91 & 0.66 & 0.91 & 0.66 & 2.10 & 0.61 & 1.27 & 0.51 \\
\hline & SAW & 0.79 & 0.64 & 0.79 & 0.61 & 1.88 & 0.59 & 1.17 & 0.48 \\
\hline & Whitened & 1.03 & 0.69 & 1.04 & 0.71 & 2.32 & 0.62 & 1.37 & 0.54 \\
\hline
\end{tabular}

Note: $\mathrm{R}$ and $\mathrm{X}$ are wave packets with the fundamental and overtone phases, respectively.

fundamental mode Rayleigh waves, as well as the first and second orbit higher mode surface waves (Table 1 and Figs 7 and 8).

The misfit values reported in Table 1 and Figs 7 and 8 are defined by the root mean squared (rms)residual (predicted value - ground truth) normalized by the rms amplitude of the 3-D CSEM trace for each wave packet. Analysing these results leads to several surprising results.

For nearly all distances and wave packets, the linear Born seismograms provided the worst fit. In fact, for the fundamental modes at most distances, on average most wave packets were fit quite poorly by the first-order Born approximation, with a mean normalized misfit greater than 1. A normalized misfit of 1 can arise, for example, when a seismogram is in phase, but has an amplitude either a factor of 2 too large or nearly zero. Alternatively, a misfit of 1 can mean the amplitude is correct, but the phase is off by approximately a quarter cycle. Both of these situations would likely be characterized as a poor fit upon visual inspection.

For the fundamental modes for both classes of models (Fig. 7a), there was minimal performance difference between PAVA and NACT, as might be expected since the cross-branch coupling terms added by NACT are expected to be small for the fundamental mode compared with overtone wave packets, which contain energy from several mode branches arriving simultaneously. The modified Born approximation (NBORN) shows a large improvement over the poor fit of the linear Born traces; however, in general they show some degradation in fit relative to the PAVA and NACT traces.

Considering all models for the overtone wave packets (Fig. 8a), all theories show a better fit than for the fundamental modes. Physically, this can be explained by the deeper sensitivity of the overtones, 


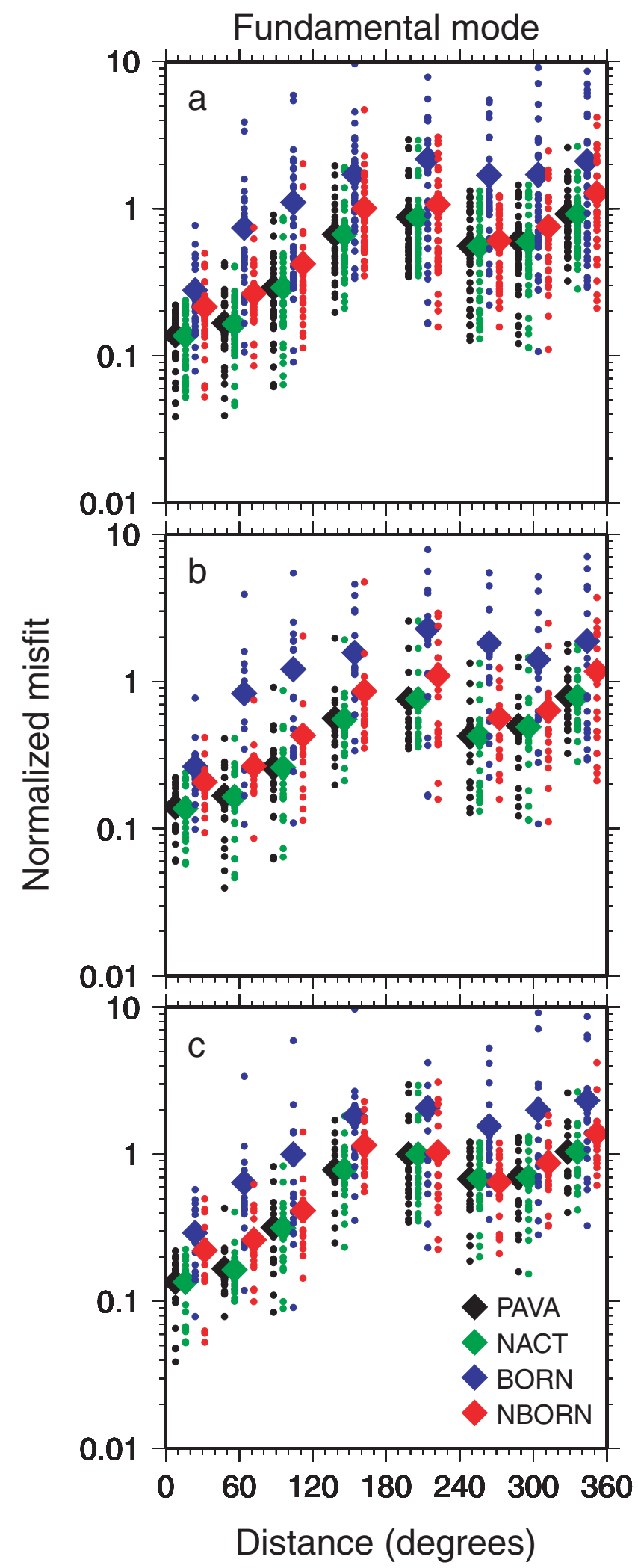

Figure 7. Normalized misfits for wave packets containing fundamental mode Rayleigh waves as a function of distance for the PAVA (black), NACT (green), linear Born (blue) and modified Born (red). All are evaluated at the same set of distances but are offset for clarity. Small dots show individual misfits, whereas the mean misfit at each distance is plotted as a larger diamond. Top panel shows the results from all models, middle panel shows only SAW24B16-like models and bottom panel shows models with whitened spectrum at degrees 13-24.

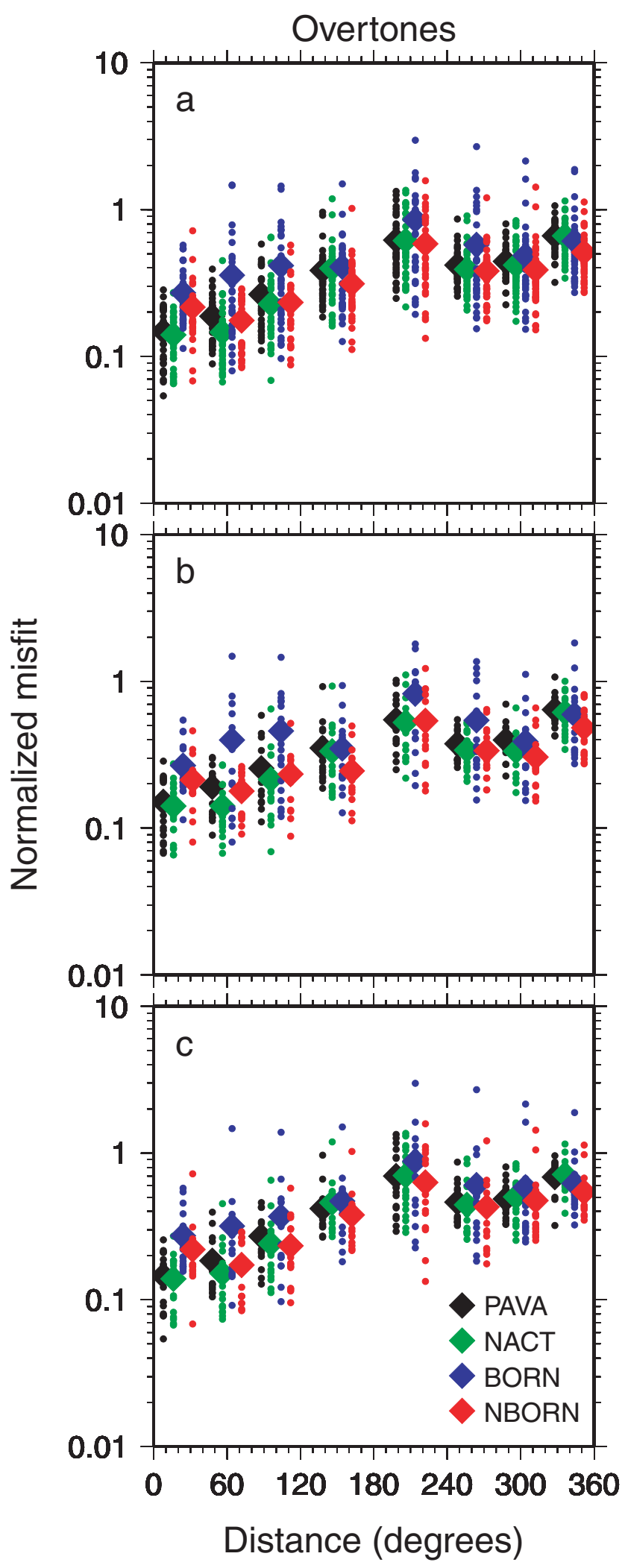

Figure 8. Same as Fig. 7 but for wave packets containing higher mode surface waveforms. 
combined with the lower amplitude of structure at greater depths in these models. This means that the overtones differ less from the reference 1-D trace and thus are easier to fit for all theories. There is a little more differentiation between PAVA and NACT, with NACT showing a moderate improvement in most distance ranges. The linear Born remains the worst fit, but there is less difference from the great-circle based approximations than in the fundamental mode case. This is likely a combination of two factors. The lower amplitude structure leads to less accumulated phase delay, which means that fewer traces will lead to a breakdown of the linear Born approximation. However, it is also likely partially because coupling between modes across dispersion branches, accounted for asymptotically in NACT but more fully in the first-order Born approximation, is more important for the overtones, which consist of many simultaneously arriving modes. When the NBORN modification is taken into account to reduce the effect of the large phase delays, NBORN is comparable with NACT for distances up to $100^{\circ}$ and the best fit for greater distances.

It is also interesting to break down the comparisons to only include the SAW24B16-like models (Figs $7 \mathrm{~b}$ and $8 \mathrm{~b}$ ) and only the whitened spectrum models (Figs $7 \mathrm{c}$ and $8 \mathrm{c}$ ). For both fundamental and overtone phases, the difference in misfit between the linear Born traces and the other theories is decreased for the whitened spectrum models relative to the SAW24B16-like models. By inspecting the values in Table 1 , we can see that there is a consistently worse fit for PAVA and NACT for the whitened spectrum models than for the SAW24B16-like models, whereas the first-order Born traces do not show a consistent difference between the two classes of models.

What is the explanation for the poor performance of the firstorder Born approximation? To understand whether the poor misfits are arising more from mismatches in phase or amplitude, we also calculated correlation coefficient values for each fundamental mode (Fig. 9a) and overtone wave packet (Fig. 9b). If the normalized misfit is close to 1 and the correlation is positive, that is, an indication that the mismatch is primarily in amplitude, whereas if the correlation is near zero or negative, the mismatch is primarily in phase. If the normalized misfit is much greater than 1 , the waveform match is poor enough, so that the correlation coefficient is less informative. For the fundamental mode, there are clearly some traces that have poor enough fits, so that the correlation is also quite poor. However, for most traces, including many with poor misfit values, the correlation remains strongly positive, indicating that the misfit values are more indicative of amplitude mismatch than phase mismatch. This is what we would expect if the breakdown of the first-order Born traces is due to a problem related to the growth of the secular term, as discussed in Sections 2 and 3.2. The paths through the models accumulate large phase delays, but the linear Born implementation of these delays leads to a breakdown due to non-conservation of energy, and the amplitudes are thus greatly overpredicted. Since the Born fits do not vary strongly between the SAW24B16-like models and the whitened models, it is apparent that the problematic phase delays are accumulated chiefly due to long wavelength structure.

\section{COMPARING WAVEFORM AND PHASE DELAY KERNELS}

The evaluations from the previous sections are all performed specifically for time domain waveform modelling. However, in many applications of 3-D finite frequency theory to surface waves, the data are secondary observables, such as phase or group delays (e.g. Ritzwoller et al. 2002; Yoshizawa \& Kennett 2004; Zhou et al.

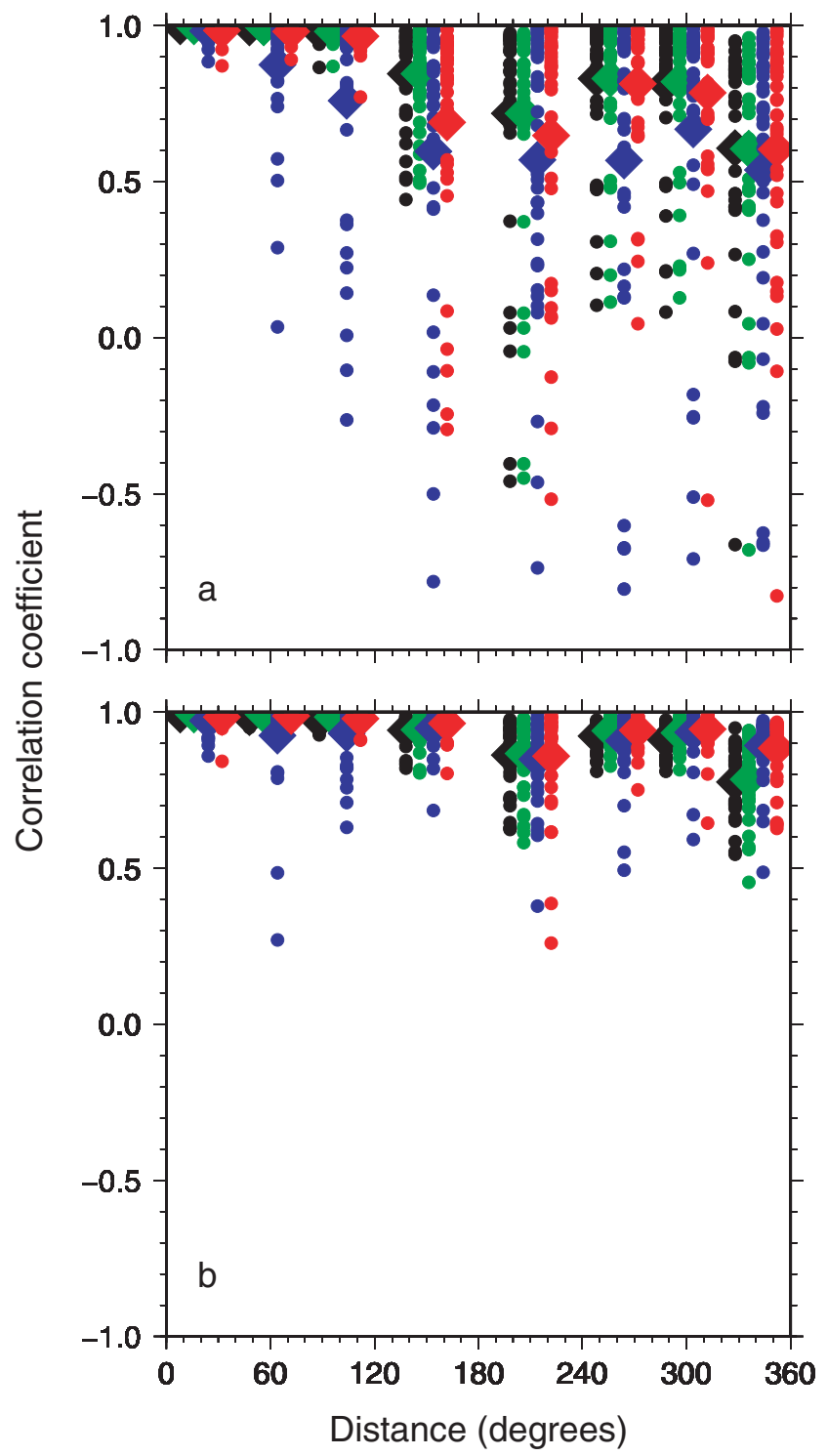

Figure 9. Same as Fig. 7 but displaying correlation coefficient rather than misfit for (a) the fundamental mode wave packets and (b) the higher mode wave packets.

2006). Although the results of Section 5 clearly indicate that at least for some frequency bands and classes of models the first-order 3-D Born time domain waveform kernels do not correctly model the waveform data, there is reason to expect that the situation will be less problematic for phase delay modelling. It is already apparent that the surface wave data maintain linearity in phase for a larger range in model parameters because PAVA waveform fits, which are implemented as linear phase perturbations, are consistently better than the 3-D Born waveform fits.

Although first-order 3-D Born phase delay kernels can be derived from the Born approximation of waveform perturbation (e.g. Zhou et al. 2004), it is important to note that there are differences in the two approaches. The results of tests of waveform studies as described above cannot be applied blindly to phase delay based approaches. The phase delay kernels are based upon the effective phase shift of an infinitesimal waveform perturbation, where the first-order Born approximation is valid by definition, and then assuming that phase delay is a linear function of model perturbation. Once again, this is 
analogous to comparing a perturbed sine wave defined by $\sin t+$ $\delta \phi \cos t$ to one defined by $\sin (t+\delta \phi)$. For small perturbations, the two are equivalent, but the results will diverge in both apparent phase and amplitude as the perturbation $\delta \phi$ becomes large.

It is possible to use the data set from Section 5 to evaluate the performance of Born phase delay kernels. We use a multitaper method (Laske \& Masters 1996) to measure relative phase delays between the 3-D CSEM traces and the 1-D reference traces. We can then convert the Born waveform kernels developed using the methodology of Capdeville (2005) to phase delay kernels appropriate for multitaper measurements using the expressions of Zhou et al. (2004).

We choose to evaluate Born phase delay kernels only for fundamental mode measurements. Because the overtones are not well isolated in time and the waveforms are an interference of several higher modes arriving simultaneously, more processing is required both to make the mode phase measurements as well as defining the kernels. This introduces several possible sources of error, which make our simple testing proposed here less informative.

We measure the phase delays on the CSEM traces through the 3-D models relative to the 1-D reference traces using the multi-taper method described by Laske \& Masters (1996). We use the first five $2.5 \pi$ prolate spheroidal tapers with a window length defined by the interval between the predicted arrival of phases with 3.5 and $4.4 \mathrm{~km} \mathrm{~s}^{-1}$ group velocities. This window isolates the fundamental mode well for the passband used here and the simplified PREM background model. We use these tapers to get five independent estimates of the complex transfer function between the 1-D and 3-D traces as a function of frequency, $T(\omega)$. If agreement with the 1-D trace were perfect, then $T(\omega)=1$, whereas a small perturbation leads to $T(\omega)=1+\delta T(\omega)$. If we assume that the 3 -D trace is sufficiently close to the reference trace (i.e. $\delta T$ is small), then we can write

$\delta T(\omega) \approx \ln T(\omega)=\delta \ln A(\omega)-\mathrm{i} \delta \phi(\omega)$,

where $\delta \phi(\omega)$ is the phase delay, and $\delta \ln A(\omega)$ is the relative amplitude anomaly. We can then measure $\delta \phi$ by

$\delta \phi(\omega)=-\operatorname{Im}(\ln (T(\omega)))$,

and we choose to use the mean of the independent taper estimates for our measured $\delta \phi$.

Following Zhou et al. (2004), we can then convert our 3-D Born time-domain waveform kernels, $\mathcal{K}(\mathbf{x}, t)$, to kernels appropriate for the multitaper measurements using the expression

$K_{\phi}(\mathbf{x}, \omega)=-\operatorname{Im}\left(\frac{\sum_{j} \mathcal{K}_{j}(\mathbf{x}, \omega) s_{j}^{*}(\omega)}{\sum_{j} s_{j}(\omega) s_{j}^{*}(\omega)}\right)$,

where $j$ is an index of the time domain tapers used, $s_{j}(\omega)$ is the $j$ th windowed 1-D reference trace transformed to the Fourier domain and $\mathcal{K}_{j}$ is the $j$ th windowed waveform kernel transformed to the Fourier domain. The predicted phase delay is then

$\delta \phi^{(\text {pre })}(\omega)=\int K_{\phi}(\mathbf{x}, \omega) \delta m(\mathbf{x}) \mathrm{d} \mathbf{x}$,

where $\delta m(\mathbf{x})$ is the shear velocity perturbation model. The phase delay is then converted from radians to seconds.

Because the corner periods of the passband for this data are 100 and $250 \mathrm{~s}$, we choose to report the results for 120,160 and $200 \mathrm{~s}$ (Fig. 10). To minimize the effects of cycle skip, we assume that the phase delay is less than $\pm \pi$ radians at a frequency of $3.5 \mathrm{mHz}$ and then unwrap the phase to higher frequencies from that point.

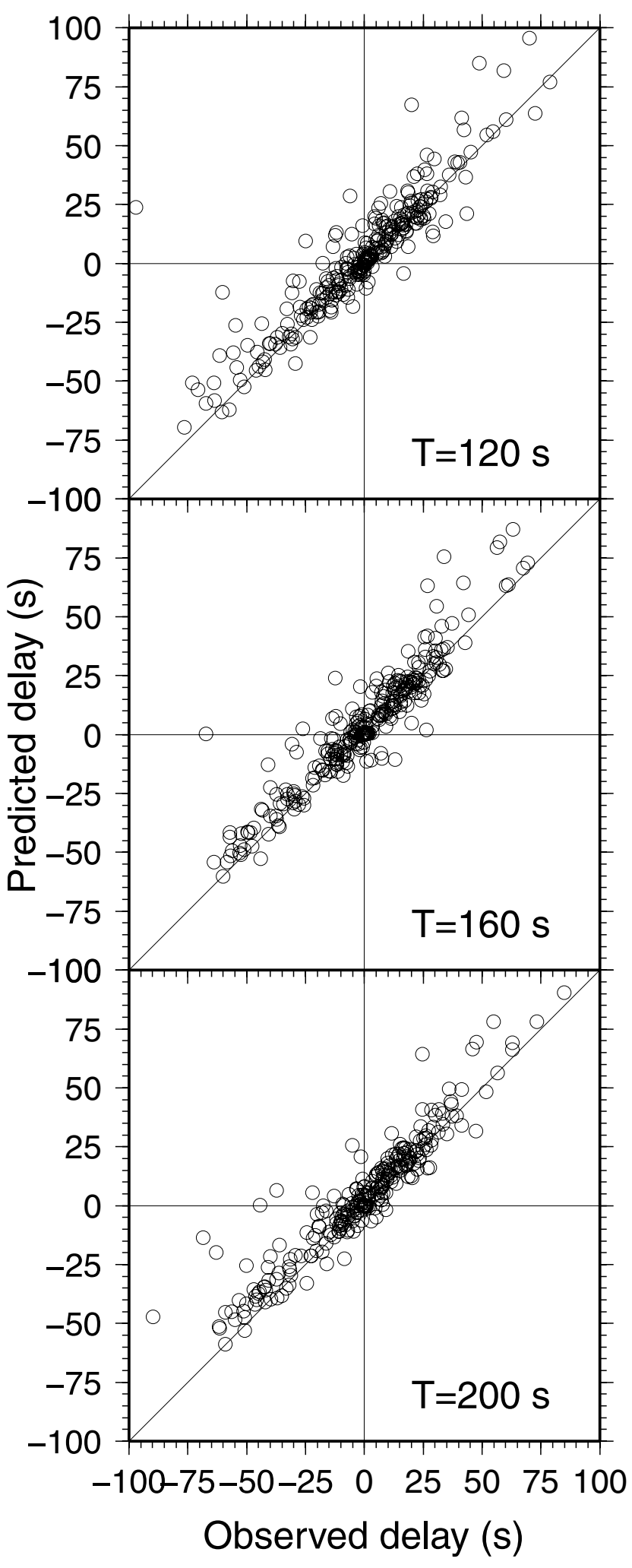

Figure 10. Fundamental mode Rayleigh wave phase delays (s) measured from 3-D CSEM traces plotted versus predictions from linear 3-D Born phase delay kernels at periods of (a) 120, (b) 160 and (c) $200 \mathrm{~s}$. 
Table 2. Born and PAVA phase delay statistics.

\begin{tabular}{lccc}
\hline Period & & Born & PAVA \\
\hline $120 \mathrm{~s}$ & Slope & 0.88 & 0.83 \\
& $R^{2}$ & 0.82 & 0.78 \\
& RMS error (s) & 12.1 & 12.8 \\
& $\chi^{2} / N$ & 1217.1 & 611.2 \\
$160 \mathrm{~s}$ & Slope & 0.96 & 0.68 \\
& $R^{2}$ & 0.88 & 0.73 \\
& $\mathrm{RMS}$ error (s) & 9.8 & 12.9 \\
& $\chi^{2} / N$ & 1034.9 & 2698.7 \\
& Slope & 0.89 & 0.48 \\
& $R^{2}$ & 0.88 & 0.66 \\
& $\mathrm{RMS}$ error (s) & 9.8 & 15.9 \\
& $\chi^{2} / N$ & 71.8 & 193.0 \\
\hline
\end{tabular}

Note: For each period, the slope and $R^{2}$ of the least-squares linear regression between observed phase delays and those predicted by Born kernels and by PAVA are shown, as well as the rms misfit and reduced $\chi^{2}$ statistic describing the fit of the predictions to the data.

There is general agreement between the measurement and prediction at all three periods, and there does not appear to be significant bias. The slopes of the best fit lines through the plots of observed versus predicted phase delay are slightly less than unity at all three periods (Table 2), suggesting a slight but likely insignificant degree of underprediction. The relationship is well-approximated by a line with $R^{2}$ values greater than 0.8 for all three periods. There is slightly more scatter at $120 \mathrm{~s}$, with a rms prediction error of $12.1 \mathrm{~s}$, compared with rms error of $9.8 \mathrm{~s}$ at both 160 and $200 \mathrm{~s}$, but the agreement is fairly good for all three periods.

Using the multitaper method, we can obtain independent estimates of the measurement error of the transfer function using a jackknife method (Thomson \& Chave 1991; Laske \& Masters 1996). This approach gives a complex valued estimate of the variance of the transfer function, $\sigma_{T}^{2}(\omega)$. We convert this to a phase delay measurement error by making the conservative assumption that the error in transfer function estimate is distributed in a circular region in the complex plane. We also make the conservative assumption for error propagation that $\operatorname{Im}(\ln (T)) \approx \operatorname{Im}(T)$, which is an overestimate for larger phase delays. We then estimate the real-valued error on the phase delay measurement by $\sigma_{\delta \phi}(\omega)=\sqrt{\left|\sigma_{T}^{2}(\omega)\right|}$. If this is an accurate estimate of the measurement error, and the Born phase delay predictions are accurate within measurement error, then we would expect a value near unity for the reduced $\chi^{2}$ statistic of the measurement error

$\frac{\chi^{2}}{N}=\frac{1}{N} \sum_{i=1}^{N}\left[\frac{\left(\delta \phi_{i}^{(\text {pre })}-\delta \phi_{i}\right)^{2}}{\sigma_{\delta \phi, i}^{2}}\right]$,

where $N$ is the number of measurements of $\delta \phi$ and $\sigma_{\delta \phi, i}$ is the estimated error of the $i$ th measurement.

For this data set we obtain very large values of $\chi^{2} / N$ of 1217.1, 1034.9 and 71.8 at 120,160 and $200 \mathrm{~s}$, respectively (Table 2). There are two possible explanations for these large values. The measurement error may be significantly underestimated by a factor of $\sqrt{\chi^{2} / N}(34.9,32.2$ and 8.5 for 120,160 and $200 \mathrm{~s})$, or the theory is incapable of matching the measurements within measurement error, particularly as we move to higher frequencies. It is possible that this estimate of error may be a significant underestimate, as it only considers the error caused by the multitaper method itself. In particular, this includes spectral leakage and inconsistencies in the effective phase delays in different portions of the time domain window chosen, as might be expected in a multimode waveform or in the presence of noise (which is not included here). Laske \& Masters (1996) found that the jackknife errors were an underestimate in their real data set and had to be multiplied by a factor of 2 for self-consistency. Real data, of course, would also include noise not present in this synthetic data as well as other potential sources of error such as errors in instrument response and the choice of method for computing reference traces in an unknown model, and thus we could expect significantly higher measurement error than in the case of the noiseless synthetics considered. These factors may be sufficient to explain the large reduced $\chi^{2}$ statistics simply as an underestimate of the true variance value that should go in the denominator of the statistic for this synthetic case with a known model. However, based on this data set, there is justification for further study of how well Born phase delay kernels are able to fit data at shorter periods within measurement error.

We can also compare the performance of the Born phase delay predictions with those from PAVA. We explicitly express the PAVA predictions of phase delay for a particular mode, in seconds, for the minor and major arc phases using the frequency perturbations from eqs (11) and (12) as

$\delta \phi_{1}^{(\mathrm{PAVA})}=\frac{\Delta}{U_{k} \omega_{k}} \delta \tilde{\omega}_{k}$,

$\delta \phi_{2}^{(\mathrm{PAVA})}=\frac{1}{U_{k} \omega_{k}}\left(2 \pi \delta \hat{\omega}_{k}-\Delta \delta \tilde{\omega}_{k}\right)$,

where, as before, $U_{k}$ is group velocity of the mode, in radians per second, and the subscripts 1 and 2 refer to minor and major arc, respectively. For the set of paths used here, the relation between observed phase delays on the synthetic seismograms and the PAVA predictions are shown in Fig. 11. For the predictions shown here, we took an additional step to make the phase delay predictions directly comparable with the multitaper measurements. Since the PAVA predictions assume the only effect of the model on the seismogram is a phase perturbation, the transfer function is simply $T(\omega)=$ $\exp \left(-\mathrm{i} \delta \phi^{(\mathrm{PAVA})}\right)$. To account for the smoothing in the spectral domain of the multi-taper estimation of the spectrum, we convolve this transfer function with the frequency domain tapers used in the measurement. Because the PAVA predicted phase delays are a smooth function of frequency, however, this step has little effect on the final prediction, except near the edges of the frequency band, where spectral leakage makes the measurements unreliable anyway.

The PAVA predictions also have generally good agreement with the measurements, although not quite as good as the Born predictions. There appears to be some increasing underprediction at longer frequencies, as the best fit lines through the data of Fig. 11 have slopes progressively less than 1 as we move to longer period (Table 2). The least-squares linear regressions of the predictions to the observations are also somewhat less well fit, with $R^{2}$ values less than those for Born at equivalent periods. The rms misfit is similar to Born at $120 \mathrm{~s}$, but worse as we move to longer periods. When we take into account the measurement error and consider the normalized $\chi^{2}$ statistic, we obtain slightly better statistics compared with Born for the shortest period. At longer periods, however, the fit is slightly worse than Born. This may be somewhat unexpected, as we showed for the waveforms that PAVA performed better than Born for long wavelength structure, and so we might expect that to mean that PAVA would also perform better at matching the lower frequency data. However, the breakdown in Born was not specifically due to the wavelength of the structure, but was actually due to the larger 


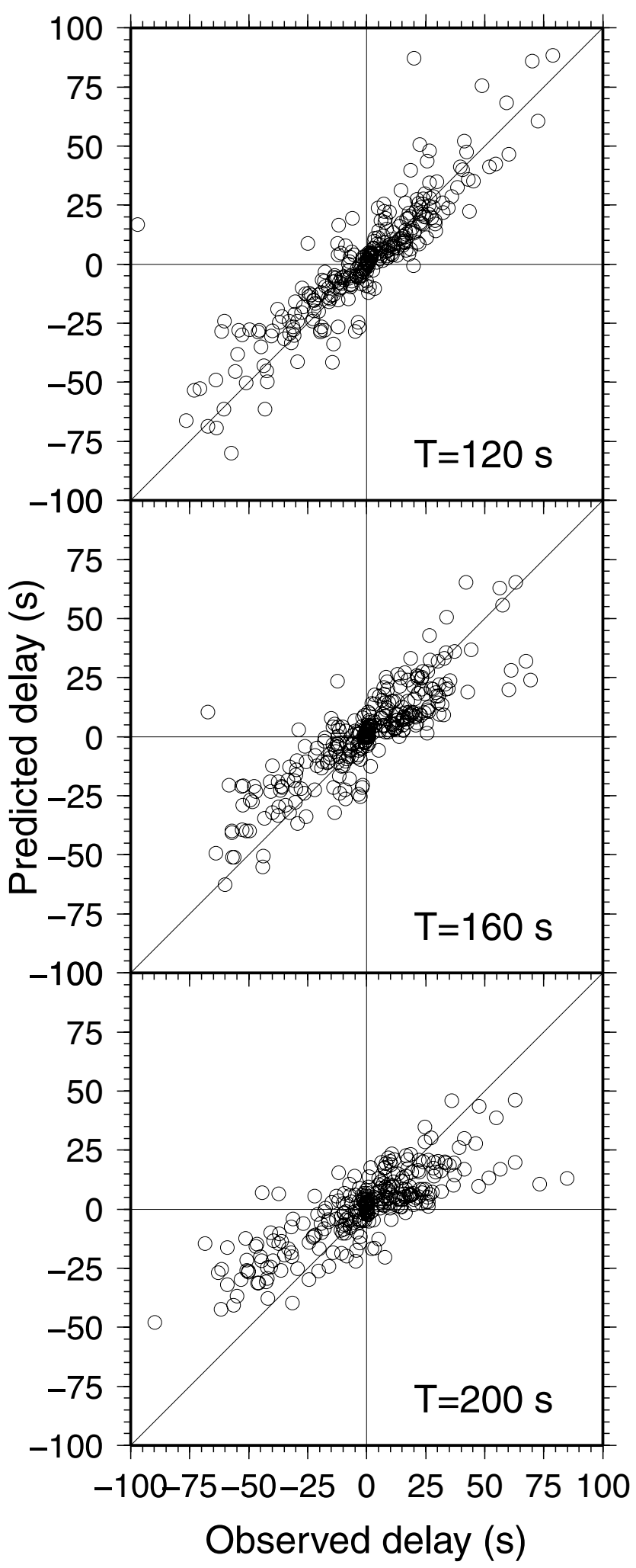

Figure 11. Same as Fig. 10 but for PAVA predictions.

cumulative phase delays due to longer wavelength structure causing a breakdown in the linearly perturbed waveforms. Converting the Born kernels to phase delay kernels avoids this shortcoming. The improved performance relative to PAVA at longer periods can likely be explained by the larger contribution of off-path structure for the longest period data.

We do not compare phase delay predictions for NACT or the modified Born approach here. This decision was made because both methods are hybrid compromises between approaches that assume linear waveform perturbations, such as the first-order Born approximation to perturbed waveforms, and methods like PAVA that assume linear phase perturbations. This makes the conversion to pure phase delay predictions a non-trivial problem. We also have little reason to suspect that NACT and NBORN would offer significant improvement in terms of a phase-delay prediction over PAVA and Born phase delay kernels, respectively, as both approaches are designed specifically to improve waveform mismatches.

\section{DISCUSSION}

As can be predicted from the assumptions that go into the method, waveform modelling based on a 3-D Born approximation performs impressively for models with small deviations from the reference model, matching numerical predictions nearly exactly. However, it is clear that the assumptions going into the approximation can be violated for realistic Earth data when starting from a 1-D reference model. It appears that for surface waveforms at periods near $100 \mathrm{~s}$ at least, the fit from the forward application of 3-D Born waveform modelling is either not sufficient for improving global mantle velocity models or requires great care in selection of the data used in the inversion.

This in no way means that 3-D Born waveform modelling does not have potential for advancing global scale modelling. The simple geometries discussed here showed that 3-D Born-based kernels offer significant improvements in accurately modelling the effect of shorter wavelength structure. The simple and computationally efficient PAVA-based non-linear modification discussed in Section 4 greatly improves the performance of the linear Born approximation by improving the modelling of the effects of the large wavelength structure, which can generate larger phase delays, and has great potential for use in global modelling in combination with very careful data selection to avoid paths where the theory breaks down. This sort of careful data selection was used in the Born waveform modelling performed by Takeuchi (2007), who used purely linear kernels. An attempt was made to reduce the potential bias introduced by discarding all data exhibiting larger phase delays by using data in multiple frequency bands. This allowed the inclusion of paths with significant accumulated structure at least for longer period data, where the total phase delay in radians is less. Even more data could be accurately modelled if a correction as in Section 4 is used, particularly for higher mode data. This is very important, as there is reason to argue that waveform modelling, which accurately treats higher mode waveforms as the interference pattern of several modes with similar group velocities, is a preferable method to the greater amount of pre-processing and potential error sources required to use higher mode phase and group delays.

This also does not directly address the gains to be made by Bornbased modelling of other seismic observables, such as body-wave cross-correlation traveltimes and surface wave group and phase delays. Section 6 suggests that the Born phase delay kernels perform better than the waveform kernels, although there may still be potentially significant errors in measurement fit. For the fundamental mode Rayleigh wave in the frequencies studied here, the Born phase delay kernels generally outperformed the predictions from the path average approximation. This merits further systematic studies 
comparing the forward performance of Born theory with other theoretical approaches to modelling phase delay measurements, such as a JWKB approximation (e.g. Dahlen \& Tromp 1998, chapter 16).

Another avenue for improving the waveform modelling using Born-based kernels is to find some way to bring the reference model closer to the real model. Based on the poor waveform fits in the realistic earth models where the average 1-D structure was known exactly, it appears that a single 1-D reference model is not sufficient for the direct application of linear Born kernels. The most straightforward way to do this is to use numerical approaches to utilize a 3-D starting model, which has been proposed for Born-based theories for various seismic observables on the global scale using an adjoint wavefield method (Tromp 2005; Liu \& Tromp 2006; Tape et al. 2007) and on a regional scale using a more traditional matrix inversion technique (Chen et al. 2007). This, however, remains a computationally expensive option. Another option is to use a Bornbased approximation calculated relative to a non-linearly modelled 1-D model for each path (Meier et al. 1997). For large regional and near-teleseismic modelling, there have also been proposals to move beyond the single scattering approximation by using an algorithm to incorporate multiple forward scattering (Friederich et al. 1993; Friederich 1999). This algorithm is somewhat more computationally intensive than the approach described in this study and requires careful reparametrization and discretization of the model for each event used, but does an excellent job of avoiding the pitfalls of the first-order Born approximation. Finally, it may also be possible to implement a Born approximation using regionalized mode calculations, analogous to non-linear crustal corrections applied in regional and global waveform modelling (Panning \& Romanowicz 2006; Marone \& Romanowicz 2007).

Finally, the greater range of validity of PAVA and NACT are explained by the implementation of linear phase rather than amplitude perturbations. This suggests that a method based on the Rytov approximation, which explicitly develops 3-D kernels based on linear phase rather than amplitude perturbations, may be a fruitful avenue. At this point, however, no Rytov-based method has been developed for a multimode setting, with attention being restricted to isolated modes like the fundamental mode surface wave (Spetzler \& Snieder 2001) and first-arriving $P$ waves, using acoustics (e.g. Woodward 1992). For practical modelling of much of the seismic waveform, a Rytov-based theory needs to be able to handle multimode settings and correctly model mode conversions, such as $P$ to $S$ conversions, and scattering between higher mode surface wave branches, which may be outside the scope of the theory.

It is important for each application of linear Born kernels, though, that some effort is made in making sure that the addition of a more computationally expensive approach is leading to actual improvement in data modelling. This study demonstrates that a blind application of the theory may lead to undesirable performance in comparison with simpler theories.

\section{CONCLUSIONS}

Although 3-D time domain waveform modelling based on the Born approximation shows promise for improving large scale seismic velocity models, it is important to carefully validate the method with numerical results. Based on simple tests comparing with waveforms calculated with coupled normal mode and spectral element method (Capdeville et al. 2002), we show that 3-D Born waveform kernels do improve modelling of waveforms perturbed by anomalies with wavelengths comparable to the first Fresnel zone compared with great-circle based approximations. However, larger wavelength and amplitude anomalies can produce large phase delays that cause the linear Born approximation to breakdown in frequency ranges of interest for global seismic modelling, whereas great-circle based methods can fit the data by perturbing the phase rather than the amplitude of the waveform.

Born-modelled waveforms can be improved by adding in higher order terms based on phase delay predicted by PAVA (Woodhouse \& Dziewonski 1984). With the simple numerical tests, we show that such a modification significantly improves the modelling of waveforms perturbed by large phase delays from long-wavelength structure, while still modelling the 3-D sensitivity effects important to correctly model the effect of shorter-wavelength structure.

We also tested all of the methods in random models with structural wavelengths consistent with the global $S$-velocity model SAW24B16 (Mégnin \& Romanowicz 2000), as well as those with enhanced structure at shorter wavelengths. We observed that waveforms calculated with the linear Born approximation had worse misfit for first- and second-orbit Rayleigh and higher mode surface waveforms than the great-circle based approximations at all distances tested greater than $20^{\circ}$. This was particularly true for fundamental modes, where the average misfit for linear Born kernels was quite poor, with rms misfits normalized by the rms amplitude of the numerical traces greater than 1 for distances larger than $60^{\circ}$. Our proposed modification to the linear Born waveform modelling, however, consistently improved the fit relative to the linear Born waveforms and produced the best waveform fits of the methods tested for the higher mode surface waveforms.

Whereas the above tests only strictly apply to time domain waveform kernels, we also tested 3-D Born phase delay kernels for multitaper measurements of fundamental mode Rayleigh waves (Zhou et al. 2004), which have been applied for global $S$-velocity modelling (Zhou et al. 2006). These appear to be much less problematic than the linear waveform kernels, as predictions generally agree with measurements on the numerical data and offer some improvement relative to predictions from the path average approximation. These predictions, however, still do not appear to match observations within measurement error. Further numerical testing of such phase delay kernels is warranted to determine what gains are to be made over other approaches, and how best to select data that are well modelled by the theory.

\section{ACKNOWLEDGMENTS}

All figures were made using GMT (Wessel \& Smith 1998). Figs 2-5 produced with help from Yuancheng Gung (Gung 2003). This work benefitted from discussions with F.A. Dahlen and Guust Nolet and from the comments of two anonymous reviewers. MPP was supported through funding by Princeton University Council of Science and Technology and NSF grants EAR 0308750, EAR 0309298 and EAR 0105387. This is BSL contribution 08-12.

\section{REFER E N CES}

Boschi, L., 2006. Global multiresolution models of surface wave propagation: comparing equivalently regularized born and ray theoretical solutions, Geophys. J. Int., 167, 238-252.

Brenders, A. \& Pratt, R., 2007. Efficient waveform tomography for lithospheric imaging: implications for realistic two-dimensional acquisition geometries and low-frequency data, Geophys. J. Int., 168, 152-170.

Capdeville, Y., 2005. An efficient Born normal mode method to compute sensitivity kernels and synthetic seismograms in the earth, Geophys. J. Int., 163(2), 639-646. 
Capdeville, Y., Chaljub, E., Vilotte, J.-P. \& Montagner, J.-P., 2002. Coupling the spectral element method with a modal solution for elastic wave propagation in global Earth models, Geophys. J. Int., 152, 34-66.

Chaljub, E., Capdeville, Y. \& Vilotte, J.-P., 2003. Solving elastodynamics in a fluid-solid heterogeneous sphere: a parallel spectral element approximation on non-conforming grids, J. Comp. Phys., 187, 457-491.

Chen, P., Jordan, T. \& Zhao, L., 2007. Full three-dimensional tomography: a comparison between the scattering-integral and adjoint-wavefield methods, Geophys. J. Int., 170, 175-181.

Dahlen, F. \& Tromp, J., 1998. Theoretical Global Seismology, Princeton University Press, Princeton, NJ.

Dahlen, F., Hung, S.-H. \& Nolet, G., 2000. Frechet kernels for finitefrequency traveltimes - I. Theory, Geophys. J. Int., 141, 157-174.

Dziewonski, A. \& Anderson, D., 1981. Preliminary reference earth model, Phys. Earth planet. Inter., 25, 297-356.

Friederich, W., 1999. Propagation of seismic shear and surface waves in a laterally heterogeneous mantle by multiple forward scattering, Geophys. J. Int., 136, 180-204.

Friederich, W., Wielandt, E. \& Strange, S., 1993. Multiple forward scattering of surface waves: comparison with an exact solution and Born singlescatternig methods, Geophys. J. Int., 112, 264-275.

Gu, Y., Dziewonski, A. \& Ekström, G., 2003. Simultaneous inversion for mantle shear velocity and topography of transition zone discontinuities, Geophys. J. Int., 154, 559-583.

Gung, Y., 2003. Lateral variations in attenuation and anisotropy of the upper mantle from seismic waveform tomography, $P h D$ thesis, University of California at Berkeley, Berkeley, CA.

Hung, S.-H., Dahlen, F. \& Nolet, G., 2001. Wavefront healing: a bananadoughnut perspective, Geophys. J. Int., 146, 289-312.

Jordan, T., 1978. Procedure for estimating lateral variations from lowfrequency eigen-spectra data, Geophys. J. R. astr. Soc., 52(3), 441455.

Laske, G. \& Masters, G., 1996. Constraints on global phase velocity maps from long-period polarization data, J. geophys. Res., 101(B7), 16059 16075.

Li, X.-D. \& Romanowicz, B., 1995. Comparison of global waveform inversions with and without considering cross-branch modal coupling, Geophys. J. Int., 121, 695-709.

Li, X.-D. \& Romanowicz, B., 1996. Global mantle shear velocity model developed using nonlinear asymptotic coupling theory, J. geophys. Res., 101(B10), 22 245-22 272.

Li, X.-D. \& Tanimoto, T., 1993. Waveforms of long-period body waves in a slightly aspherical earth model, Geophys. J. Int., 112, 92-102.

Liu, Q. \& Tromp, J., 2006. Finite-frequency kernels based upon adjoint methods, Bull. seism. Soc. Am., 96, 2383-2397.

Manquering, H. \& Snieder, R., 1995. Surface-wave mode coupling for efficient forward modelling and inversion of body-wave phases, Geophys. J. Int., 120, 186-208.

Marone, F. \& Romanowicz, B., 2007. Non-linear crustal correction in high-resolution regional waveform seismic tomography, Geophys. J. Int., 170(1), 460-467.

Meier, T., Lebedev, S., Nolet, G. \& Dahlen, F., 1997. Diffraction tomography using multimode surface waves, J. geophys. Res., 102(B4), 8255-8267.

Mégnin, C. \& Romanowicz, B., 2000. The 3-D shear velocity structure of the mantle from the inversion of body, surface, and higher mode waveforms, Geophys. J. Int., 143, 709-728.

Mochizuki, E., 1986. The free oscillations of an anisotropic and heterogeneous Earth, Geophys. J. R. astr. Soc., 86, 167-176.

Montelli, R., Nolet, G., Dahlen, F., Masters, G., Engdahl, E. \& Hung, S.-H., 2004. Finite-frequency tomography reveals a variety of plumes in the mantle, Science, 303(5656), 338-343.

Montelli, R., Nolet, G., Dahlen, F. \& Masters, G., 2006. A catalogue of deep mantle plumes: new results from finite-frequency tomography, Geochem. Geophys. Geosys. (G3), 7, Q11007.

Panning, M. \& Romanowicz, B., 2006. A three-dimensional radially anisotropic model of shear velocity in the whole mantle, Geophys. J. Int., 167, 361-379.

Phinney, R. \& Burridge, R., 1973. Representation of the elastic-gravitational excitation of a spherical earth model by generalized spherical harmonics, Geophys. J. R. astr. Soc., 34, 451-487.

Ritzwoller, M., Shapiro, N., Barmin, M. \& Levshin, A., 2002. Global surface wave diffraction tomography, J. geophys. Res., 107(B12), doi:10.1029/2002JB001777.

Romanowicz, B., 1987. Multiplet multiplet coupling due to lateral heterogeneity: asymptotic effects on the amplitude and frequency of the Earth's normal modes, Geophys. J. R. astr. Soc., 90(1), 75-100.

Romanowicz, B. \& Snieder, R., 1988. A new formalism for the effect of lateral heterogeneity on normal modes and surface waves, II: general anisotropic perturbation, Geophys. J. Int., 93, 91-99.

Romanowicz, B., Panning, M., Gung, Y. \& Capdeville, Y., 2008. On the computation of long period seismograms in a 3D Earth using normal mode based approximations, Geophys. J. Int., 175, 520-536.

Spetzler, J. \& Snieder, R., 2001. The effect of small-scale heterogeneity on the arrival time of waves, Geophys. J. Int., 145, 786-796.

Spetzler, J., Trampert, J. \& Snieder, R., 2001. Are we exceeding the limits of the great circle approximation in global surface wave tomography?, Geophys. Res. Lett., 28(12), 2341-2344.

Takeuchi, N., 2007. Whole mantle SH velocity model constrained by waveform inversion based on three-dimensional Born kernels, Geophys. J. Int., 169, 1153-1163.

Tanimoto, T., 1984. A simple derivation of the formula to calculate synthetic long-period seismograms in a heterogeneous Earth by normal mode summation, Geophys. J. R. astr. Soc., 77, 275-278.

Tape, C., Liu, Q. \& Tromp, J., 2007. Finite-frequency tomography using adjoint methods: methodology and examples using membrane surface waves, Geophys. J. Int., 168, 1105-1129.

Thomson, D. \& Chave, A., 1991. Jackknife error estimates for spectra, coherences and transfer functions, Chap. 2 in Advances in Spectrum Analysis and Array Processing, Vol. 1, pp. 58-113, ed. Chaykin, S., Prentice Hall, Englewood Cliffs, NJ.

Tromp, J., Tape, C. \& Lin, Q., 2005. Seismic tomography, adjoint methods, time-reversal and banana-doughnut kernals, Geophys. J. Int., 160, 195216.

Wessel, P. \& Smith, W., 1998. New, improved version of generic mapping tools released, EOS, Trans. Am. geophys. Un., 79, 579.

Woodhouse, J., 1980. The coupling and attenuation of nearly resonant multiplets in the Earth's free oscillation spectrum, Geophys. J. R. astr. Soc., 61, 261-283.

Woodhouse, J. \& Dziewonski, A., 1984. Mapping the upper mantle: three dimensional modeling of Earth structure by inversion of seismic waveforms, J. geophys. Res., 89, 5953-5986.

Woodhouse, J. \& Girnius, T., 1982. Surface waves and free oscillations in a regionalized Earth model, Geophys. J. R. astr. Soc., 68, 653-673.

Woodward, M., 1992. Wave-equation tomography, Geophysics, 57(1), 1526.

Yoshizawa, K. \& Kennett, B., 2004. Multimode surface wave tomography for the Australian region using a three-stage approach incorporating finite frequency effects, J. Geophys. Res., 109, doi:10.1029/2002JB002254.

Zhao, L. \& Jordan, T., 2006. Structural sensitivities of finite-frequency seismic waves: a full-wave approach, Geophys. J. Int., 165, 981-990.

Zhao, L., Jordan, T. \& Chapman, C., 2000. Three-dimensional Frèchet differential kernels for seismic delay times, Geophys. J. Int., 141, 558576.

Zhou, Y., Dahlen, F. \& Nolet, G., 2004. Three-dimensional sensitivity kernels for surface wave observables, Geophys. J. Int., 158, 142-168.

Zhou, Y., Nolet, G., Dahlen, F. \& Laske, G., 2006. Global upper-mantle structure from finite-frequency surface-wave tomography, J. geophys. Res., 111, B04304, doi:10.1029/2005JB003677.

\section{APPENDIX: BORN APPROXIMATION IN NORMAL MODE FORMALISM}

We consider how to describe the first-order Born approximation in a normal mode formalism. The normal modes, or free oscillations of the Earth, are the solutions to the eigenproblem defined by eq. (1). In 
a spherically symmetric reference model, where the Earth's rotation and ellipticity are neglected, these solutions turn out to be an infinite series of modes described by three integers: $(n, l, m)$, where $n$ is the radial order, $l$ is the angular order and $m$ is the azimuthal order $(-l \leq m \leq l)$. In the spherically symmetric Earth, the modes are degenerate for a given combination of $l$ and $n$, and we can choose to combine them into a single index $k$. For each multiplet $k$, there are $2 l+1$ singlets corresponding to different values of $m$, all having the same frequency $\omega_{k}$. These are the modes used in the definition of the reference seismogram in eq. (7).

Using first-order perturbation theory (e.g. Woodhouse 1980; Tanimoto 1984), a small perturbation to the reference model introduces coupling between the different modes, and following $\mathrm{Li}$ \& Tanimoto (1993), we can write the perturbed seismogram for a particular component of motion using a splitting matrix notation as

$u(t)=\operatorname{Re} \sum_{i j} R_{i} \exp \left\{\mathrm{i} t\left[\omega_{i} \delta_{i j}+\frac{Z_{i j}}{\left(\omega_{i}+\omega_{j}\right)}\right]\right\} S_{j}$,

where $i$ and $j$ are mode indices combining multiplet index $k$ and singlet index $m$, respectively, and $\delta_{i j}$ is the Kronecker delta. $S_{j}$ and $R_{i}$ are the source and receiver vectors for singlet $j$ and $i$, respectively, as defined in terms of linear combinations of mode eigenfunctions and moment tensor elements and generalized spherical harmonics (Phinney \& Burridge 1973) by Woodhouse \& Girnius (1982). $Z_{i j}$ is the splitting matrix defining the coupling of the modes caused by the model perturbation.

The splitting matrix elements can be approximated (e.g. Woodhouse 1980; Romanowicz 1987; Li \& Tanimoto 1993) by the integral

$Z_{i j}=Z_{k k^{\prime}}^{m m^{\prime}}=2 \omega_{k k^{\prime}} \int_{\Omega} \delta \omega_{k k^{\prime}}(\theta, \phi) Y_{l}^{m *}(\theta, \phi) Y_{l^{\prime}}^{m^{\prime}}(\theta, \phi) \mathrm{d} \Omega$,

where the integral is over the unit sphere, $\omega_{k k^{\prime}}=\left(\omega_{k}+\omega_{k^{\prime}}\right) / 2, Y_{l}^{m}$ is a fully normalized spherical harmonic of angular order $l$ and azimuthal order $m$ and the asterisk represents complex conjugation. The perturbations to the spherically symmetric reference model parameters enter the expression through $\delta \omega_{k k^{\prime}}$, given by

$$
\begin{aligned}
\delta \omega_{k k^{\prime}}(\theta, \phi)= & \frac{1}{2 \omega_{k k^{\prime}}}\left[\int_{0}^{a} \delta \mathbf{m}(r, \theta, \phi) \cdot \mathbf{M}_{k k^{\prime}}(r) r^{2} \mathrm{~d} r\right. \\
& \left.-\sum_{d} r_{d}^{2} h_{d}(\theta, \phi) H_{k k^{\prime}}^{d}\right]
\end{aligned}
$$

where $a$ is the radius of the Earth, $\delta \mathbf{m}$ represents volumetric perturbations to the density and elastic coefficients, $r_{d}$ and $h_{d}$ are the radius and perturbation, respectively, of the $d$ th internal discontinuity and $\mathbf{M}_{k k^{\prime}}$ and $H_{k k^{\prime}}^{d}$ are the appropriate sensitivity kernels for volumetric and discontinuity perturbations, respectively. These kernels are defined for isotropic perturbations by Woodhouse (1980), for radially anisotropy by Li \& Romanowicz (1996) and can also be defined for more general anisotropy (e.g. Mochizuki 1986; Romanowicz \& Snieder 1988). For the case $k=k^{\prime}$, the above expression defines $\delta \omega_{\text {local }}$, as used in expressions (11) and (12).

Eq. (A1) is an expression of the Born approximation, but examination quickly shows that the linear perturbation is contained within the exponential expression and therefore will not be linear for the perturbations to the time domain waveforms. Additionally, eqs (A2) and (A3) show that implementing this expression directly requires integration over the volume of the Earth for each possible mode combination. This makes implementation of eq. (A1) in inverse approaches problematic for time domain waveform inversion and computationally challenging for any application.
Because (A1) has the splitting matrix inside the exponential term, it acts like a phase perturbation and produces a non-linear effect on $u(t)$. To define the first-order linear Born approximation appropriate for time-domain waveforms, we must return to linearized version of eq. (A1) and thus remove $Z_{i j}$ from the exponential as

$u(t)=u_{0}(t)+\delta u_{\text {Born }}(t)$,

where $u_{0}$ is the reference trace from eq. (7), and

$\delta u_{\text {Born }}(t)=\operatorname{Re} \sum_{k k^{\prime}} A_{k k^{\prime}} \frac{\exp \left(\mathrm{i} \omega_{k} t\right)-\exp \left(\mathrm{i} \omega_{k^{\prime}} t\right)}{\omega_{k}^{2}-\omega_{k^{\prime}}^{2}}$,

where

$A_{k k^{\prime}}=\sum_{m m^{\prime}} R_{k}^{m} Z_{k k^{\prime}}^{m m^{\prime}} S_{k^{\prime}}^{m^{\prime}}$.

Using this expression, we can explicitly see that this is indeed a short-time approximation $\left(t \ll 2 \pi /\left\|Z_{i j} /\left(\omega_{i}+\omega_{j}\right)\right\|\right)$. This is because a secular term must be introduced into the time dependence term on the right-hand side of eq. (A5) when we consider coupling of modes with the same frequency (e.g. within a multiplet). This arises because we must consider the limit as $\omega_{k} \rightarrow \omega_{k^{\prime}}$, leading to an expression proportional to $\left[i t \exp \left(\mathrm{i} \omega_{k} t\right)\right] /\left(2 \omega_{k}\right)$. This term will grow unbounded for large times, with breakdown occurring earlier as the magnitude of $Z_{i j}$ increases. This describes exactly why PAVA outperformed the first-order Born approximation in the case discussed in Section 3.2, as PAVA keeps the perturbation term in the exponential rather than performing the linearization, which produces the secular term requiring the short time approximation.

In the development of NACT, Li \& Romanowicz (1995) proposed to increase the validity range of eq. (A4) by applying a frequency shift to each mode in eq. (A1) prior to the linearization step in such a way as to reduce the magnitude of the perturbations in $Z_{i j}$. As an example, we could consider a waveform made up of surface waves and higher mode waveforms arriving after propagating along the minor arc. The appropriate frequency shift in this case would be the minor arc frequency shift $\delta \tilde{\omega}$, and we can make the following substitutions into eq. (A1),

$\tilde{\omega}_{i}=\omega_{i}+\delta \tilde{\omega}_{i}$

$\tilde{Z}_{i j}=Z_{i j}-2 \omega_{i} \delta \tilde{\omega}_{i} \delta_{i j}$

which leaves eq. (A1) unchanged, because we are simply removing a term from the diagonal of the splitting matrix and adding it to the reference frequency.

Upon performing the linearization using this approach, we obtain

$$
\begin{aligned}
u(t)= & \operatorname{Re}\left[\sum_{k} A_{k} \exp \left(\mathrm{i} \tilde{\omega}_{k} t\right)+\delta u_{2}\right], \\
\delta u_{2}= & \sum_{k}\left[-\mathrm{i} t \delta \tilde{\omega}_{k} A_{k} \exp \mathrm{i} \tilde{\omega}_{k} t\right. \\
& \left.+\sum_{k^{\prime} \in \Gamma_{k}} A_{k k^{\prime}} \frac{\exp \left(\mathrm{i} \tilde{\omega}_{k} t\right)-\exp \left(\mathrm{i} \tilde{\omega}_{k^{\prime}} t\right)}{\left(\omega_{k}+\omega_{k^{\prime}}\right)\left(\tilde{\omega}_{k}-\tilde{\omega}_{k^{\prime}}\right)}\right],
\end{aligned}
$$

where $\Gamma_{k}$ is the subset of modes with frequency greater than or equal to $\omega_{k}$, and

$A_{k}=\sum_{m} R_{k}^{m} S_{k}^{m}$.

For NACT, further steps are then taken to limit the calculation of $A_{k k^{\prime}}$ to the great circle path using asymptotic expansions of the associated Legendre functions in the spherical harmonics and the 
stationary phase approximation. Romanowicz et al. (2008) discuss PAVA and NACT, as well as higher-order asymptotic approximations in more detail and numerically evaluate their performance. Eq. (A9), however, shows the important points that lead to an improvement over the first-order Born approximation. The first term on the right-hand side of eq. (A9) is equivalent to the PAVA expression (eq. 8) for a minor arc phase expressed with only a frequency shift, rather than a great circle average frequency shift and an apparent epicentral distance shift. The last term in eq. (A10) is nearly equivalent to the standard Born approximation (eq. A4) but evaluated with perturbed mode frequencies. The first term in $\delta u_{2}$ results from the modification term going from $Z_{i j}$ to $\tilde{Z}_{i j}$, and we can see that it functions to remove the linearized contribution of the PAVA frequency shift from the linear Born term. 